\title{
Molecular interactions behind the self-assembly and microstructure of mixed sterol organogels
}

\author{
Georgios Dalkas*1, Andrew B. Matheson², Hugh Vass², Andrei Gromov², Gareth O. \\ Lloyd $^{4}$, Vasileios Koutsos ${ }^{5}$, Paul S. Clegg², Stephen R. Euston ${ }^{1}$
}

1- School of Engineering and Physical Sciences, Institute of Mechanical Process and Energy Engineering, John Muir Building, Heriot-Watt University, Edinburgh

2- School of Physics and Astronomy, University of Edinburgh, James Clerk Maxwell Building, Edinburgh

3- EaStChem, School of Chemistry, University of Edinburgh, Edinburgh

4- School of Engineering and Physical Sciences, Institute of Chemical Sciences, William Perkin Building, Heriot-Watt University, Edinburgh

5- School of Engineering, Institute for Materials and Processes, University of Edinburgh, Sanderson Building, Edinburgh

* Correspondence to: Georgios Dalkas, Institute of Mechanical, Process \& Energy Engineering, School of Engineering \& Physical Sciences, Heriot-Watt University, EH14 4AS, Edinburgh, United Kingdom. E-mail: G.Dalkas@hw.ac.uk

\footnotetext{
Abstract

In this work, we have employed docking and atomistic molecular dynamics (MD) simulations supported by complementary experiments using atomic force microscopy, rheology and spectroscopy to investigate the self-assembled structure of $\beta$-sitosterol and $\gamma$-oryzanol
} 
molecules into cylindrical tubules in a non-aqueous solvent. Docking models of several phytosterols, including sitosterol, with oryzanol and other sterol-esters demonstrate that for systems to form tubules, the phytosterol sterane group must be stacked in a wedge shape with the esters sterane group, and a hydrogen bond must form between the hydroxyl group of the phytosterol and the carbonyl group of the ester. Molecular dynamics of the self-assembled structure were initiated with the molecules in a roughly cylindrical configuration, as suggested from previous experimental studies, and the configurations were found to be stable during 50 ns simulations. We performed MD simulations of two tubules in proximity to better understand the aggregation of these fibrils and how the fibrils interact in order to stick together. We found that an interfibril network of non-covalent bonds, in particular van der Waals and $\pi-\pi$ contacts, which is formed between the ferulic acid groups of oryzanol through the hydroxyl, methoxy and aromatic groups, is responsible for the surface-to-surface interactions between fibrils; an observation supported by molecular spectroscopy. We believe these interactions are of primary importance in creating a strong organogel network.

\section{Keywords}

Oleogels, phytosterols, self-assembly modelling, docking simulations, molecular dynamics, atomic force microscopy, rheology

\section{Introduction}

In recent years, edible oil structuring has received considerable research interest from the scientific community working in the area of food formulation. Much of this interest is linked to the possibility of using structured oil in the development of products with an improved nutritional profile, such as low in saturated fats and high in mono and/or poly unsaturated fatty 
acids. Traditionally, edible oil products that contain polyunsaturated oils, such as margarines and spreads, are solidified by adding a proportion of saturated fat (crystalline hard stock) that gives solid structure. Saturated fatty acids in food raise blood cholesterol level, which in turn has been identified as a risk factor for heart disease. An alternative method for structuring unsaturated triglyceride oils is the formation of so-called oleogels. These are gels where a liquid lipid phase is trapped in a thermo-reversible gel network formed by the self-association of low concentrations of oleogelator molecules. ${ }^{1}$ Oleogels also have potential applications in the reduction of oil migration in high fat products, controlled release of neutraceuticals and the structuring of water-in-oil emulsions. ${ }^{1}$ A number of potential oleo-gelators have been identified including mixtures of phytosterols and sterol esters. The use of phytosterols as oleogelators is particularly appealing as these have also been linked to cholesterol lowering properties, and have been incorporated into some spreads for this reason. ${ }^{2}$

The use of mixtures of phytosterols and the sterol ester $\gamma$-oryzanol as structuring agents that show synergistic gelling functionality has received a great deal of interest from researchers over the past several years. $\gamma$-oryzanol is extracted from rice bran oil and displays antioxidant activity which can be attributed to its ferulic acid group. ${ }^{3} \gamma$-oryzanol is a mixture of several ferulate (4hydroxy-3-methoxy cinnamic acid) esters of triterpene alcohol and plant sterols (Scheme 1). ${ }^{4}$ In triglycerides, equimolar mixtures of the sterol $\beta$-sitosterol and sterol ester $\gamma$-oryzanol selfassociate to gel the oil, although the individual components cannot. The mechanism for this is the formation of elongated hollow tubules with a diameter of $\approx 10 \mathrm{~nm}$ and a wall thickness of $\approx 0.8 \mathrm{~nm} .{ }^{5}$ The self-association mechanism is believed to proceed via a sitosterol-oryzanol dimer, where the sterane cores of the two molecules align through dispersion interactions facilitated by the large, relatively flat surface of the cores, with the dimer stabilised by a single hydrogen bond. ${ }^{6,7}$ The structure of the dimer is such that it adopts a wedge shape, then the stacking of the dimers results in the formation of a tubule which twists in a helical fashion..$^{5-7}$ 
So far the presence of the hydroxyl on the sterol, the carbonyl on the ester, and similar solubilities for both materials have been identified as the only pre-requisites for tubule formation to take place. ${ }^{8}$

A number of sterols other than $\beta$-sitosterol have also been shown to form oleogels with oryzanol, but alternatives to oryzanol cannot be identified as easily because the number of commercially available sterol esters is limited. Although there is no reason to suggest oryzanol is unique in its ability to form oleogels with sterols, attempts to make an oleogel with, for example, sitosterol and sitosterol linoleate have not been successful. ${ }^{5}$ Bot et al. ${ }^{5}$ believe this is because the long chain fatty acid makes the sterol ester too soluble in the oil phase hence illustrating the delicate balance of structural features that allow tubule formation. Exploitation of oleo-gelation in food systems will require that we understand the fundamentals of the selfassociation mechanism. Only then can we design optimised oleo-gelling systems for foods that have reduced saturated fats, but also the desired organoleptic properties. To achieve this a thorough exploration of the physico-chemical energy balances involved in the mechanism of oleogelation and the structure of the tubules is required, allowing a more rational selection of oleogelator candidate molecules, screening their properties and interactions before any de novo synthesis of alternative improved gelators is required. Modelling the development of gelators into fibrous structures is a very important and challenging task, and can provide significant insights to chemists and biologists. Molecular dynamic (MD) simulations have been widely used to study self-assembly pathways of low-molecular-weight gelators (LMWGs), such as peptides, ureas or plant sterols. ${ }^{9-13}$ However, formation of tubules in solution on such large size systems using all-atom MD simulations can take place over tens or hundreds of nanoseconds, which increases the computational costs. Hence, a preassembled structure is required, which is usually proposed on the basis of experimental studies. Therefore, MD simulations are carried out on a pre-constructed tubular system consisting of sufficient gelator molecules to build a 
small part of a complete fibre, and subsequently the stability or any structural modifications and interactions between the structurants during the simulation time can be investigated, and the dominant contributions to the self-assembly system identified. ${ }^{12,14,15}$

In this study we report for the first time, an in silico, method for screening sterol based oleogelators. This method, based on the widely used AUTODOCK molecular docking program, ${ }^{16}$ allows us to screen sterol/sterol-ester combinations on their ability to form a dimer stabilised by a hydrogen bond, a feature we believe critical to oleogelating capacity. This methodology can be used not only on available sterols and sterol esters, but also on molecules that are not readily available and can subsequently be synthesised if they are identified to be potent oleogelators. This methodology has been applied to a number of sterols and sterol esters related to sitosterol and oryzanol, and verified by experimental testing of selected combinations. ${ }^{12,13}$ We have shown recently by using AFM that the tubules further associate into bundles, and then bifurcate and branch to form a network gel trapping the oil phase ${ }^{17} \mathrm{MD}$ simulations were employed in an attempt to gain further insight into the structure of the tubules and how the tubules which make up the gel structure interact so as to aggregate into bundles. The origin of the bundle formation is not clear yet, however, it has been suggested from molecular modelling and SANS that when the sterol and sterol ester form a dimer and subsequently stack into a tubule, the ferulate group of oryzanol protrudes out from the tubule surface. ${ }^{18}$ We hypothesise that fibril crosslinking arises from interactions of ferulate groups on the oryzanol on separate tubules. To test this hypothesis, we have used cholesteryl hemisuccinate (CHEMS) in place of oryzanol where the ferulate moiety of oryzanol is replaced with a carboxylic group (Scheme 1), and we have carried out docking and MD simulations of CHEMS with sitosterol. Importantly, rheology and atomic force microscopy (AFM) were used to support the computational findings. 
a)

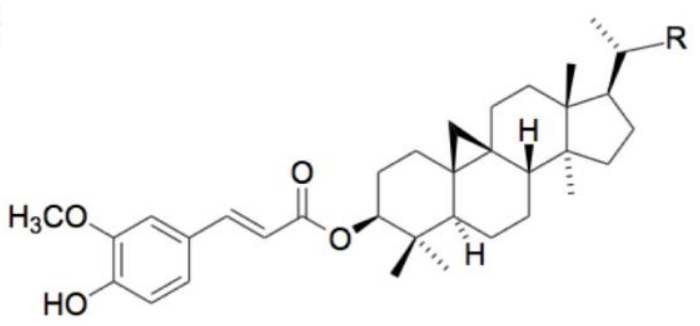<smiles>C=C(CC)C(C)C</smiles><smiles>[CH2+]CC=C(C)C</smiles>

24-methylenecycloartenyl ferulate cycloartenyl ferulate<smiles>[R]C(C)[C@H]1CC[C@H]2[C@@H]3CC=C4C[C@@H](OC(=O)/C=C/c5ccc(O)c(OC)c5)CC[C@]4(C)[C@H]3CC[C@]21C</smiles>

b)

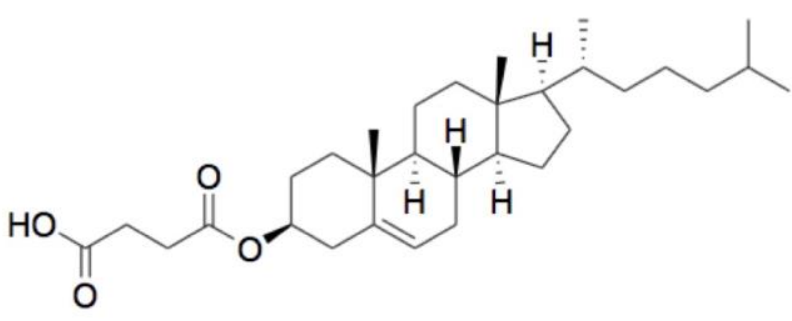

cholesteryl hemisuccinate c)

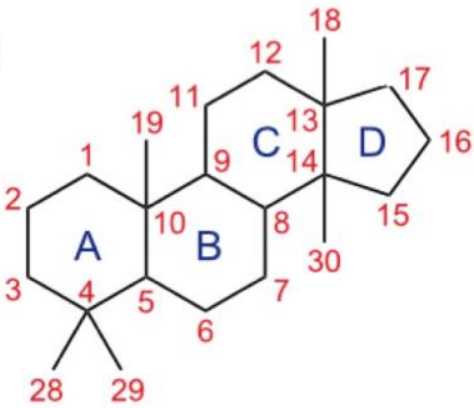

Steroid ring system

Scheme 1. a) Chemical structures of the major components of $\gamma$-oryzanol and b) chemical structure of cholesteryl hemisuccinate (CHEMS). c) The ABCD steroid ring system with atom numbering.

\section{Experimental Section}

\section{Computational methods}

Docking calculations. The 3D models of sterols and sterol esters were generated from SMILES representation using the program OMEGA $2.5^{19}$ and Gasteiger charges were applied using AutoDockTools 1.5.6. ${ }^{16}$ The search space was defined by a grid box centred on oryzanol compounds with 80 points of $0.375 \AA$ spacing in each dimension. For each complex, 100 docking rounds were calculated with AutoDock $4.2^{16}$ using the Lamarckian genetic algorithm 
with the default parameters of AutoDock. The maximum number of energy evaluations was set to $5 \times 10^{6}$, and the solvent dielectric constant was set to 2.0. The value of the solvent dielectric constant reflects the triglyceride of the solvent in the oleogelating systems. The resulting docked conformations were clustered using a tolerance of $2.0 \AA$ and the most populated cluster with the highest free energy of binding $\Delta \mathrm{G}$ was selected.

Structure preparation of the tubules. We have employed docking and MD simulations so as to define the initial tubule structures. The systems we have used for the MD simulations are the mixtures of sitosterol with either oryzanol or CHEMS. An efficient approach for obtaining the initial layer of the tubular structure of sitosterol and oryzanol has been described in our recent study. ${ }^{12}$ This fragment- and knowledge-based docking approach was also used in this study with slight adaptation so as to obtain tubular structures with six layers. The approach we used to build the sitosterol-oryzanol tubule six-layer model is described below. The docked sitosterol-oryzanol dimer with the lowest binding energy, produced using the AUTODOCK protocol described above, was used as the starting building block of the model. This dimer was one where a hydrogen bond between the hydroxyl group of the sitosterol and the carbonyl group of the oryzanol was formed. By using docking simulations, 19 dimers were docked radially on a plane with the ferulate group of oryzanol sticking out from the surface, forming a helix-like structure. The starting radius of the structure was $\sim 45 \AA$, which is in good agreement with experimental studies, as several studies reported that mixtures of oryzanol and sitosterol were found to self-assemble into elongated hollow tubular structures with an average radius of $\sim 5$ nm. ${ }^{8,20,21}$ Subsequently, the model was subjected to energy minimisation using the AMBER16 program. ${ }^{22}$ The initial structure was first minimised using 5000 steps of steepest descent minimisation followed by 5000 steps of conjugate gradient minimisation (details of the force field parameterisation are given below). 
Final structure. The second layer, which was identical to the first layer, was placed above the first layer with a distance between the two layers taken to be around $5 \AA$, and the distance between the last molecule of the first layer and the first molecule of the second layer to be around $5 \AA$ A. Finally, a total of 6 layers were placed along the fiber axis to define the complete structure. The complete tubule contained two hundred and thirty (230) sitosterol and oryzanol molecules in a 1:1 ratio. The system energy was minimised using 5000 steps of steepest descent minimisation followed by 5000 steps of conjugate gradient minimisation and then solvated in a box containing decane molecules with a minimum extension of $10 \AA$ from the solute, using the XLEaP module of AMBER16. Scattering experiments showed that the morphology of the sitosterol-oryzanol tubules is preserved in different organic phases ${ }^{21}$ which means that the experimentally observed tubule structures in sunflower oil should be comparable with our MD simulations performed in decane. The total charge of the system was neutral, and periodic boundary conditions were used in the simulations.

In order to run MD simulations of two tubules, we have placed two single tubules in a simulation box using the XLEaP, with the distance between the two tubules taken to be around $10 \AA$.

Molecular dynamics simulations. The AMBER v16 package was used for simulation of the systems. Force-field parameters for all the compounds were prepared using the Antechamber package, ${ }^{23}$ and the AM1-BCC atomic charges. The $\mathrm{ff} 4 \mathrm{SB}^{24}$ force field parameters and the General-AMBER force field $(\mathrm{GAFF})^{23}$ were applied to the structure atoms using the XLEaP module of AMBER16. After equilibration of the systems (details in the Supporting Information), a production run of $50 \mathrm{~ns}$ of molecular dynamics was simulated at constant temperature and pressure. Analysis was conducted on the equilibrated part of the trajectories using the CPPTRAJ module ${ }^{25}$ of AmberTools v16, and VMD 1.9. $2^{26}$ was used to visualize the 
trajectories and prepare the figures. CPPTRAJ was used to find and track hydrogen bonds over the course of the trajectories, using for both distance and angle cut-offs the default values for hydrogen bonds of CPPTRAJ module.

\section{Experimental methods}

Oryzanol and sitosterol were provided by Unilever and all other materials were purchased from Sigma Aldrich. All materials were used as supplied without any additional purification.

Gels were prepared by dissolving sterol and sterol ester at a 1:1 molar ratio in sunflower oil at $\sim 90{ }^{\circ} \mathrm{C}$, such that each sample was $15 \%$ gelator by mass. For rheology measurements, molten gels were poured directly onto the baseplate of a TA instruments AR2000 rheometer. A crosshatched plate geometry was used, with a gap of $1 \mathrm{~mm}$. An oscillatory stress of $170 \mathrm{~Pa}$ was applied at $10 \mathrm{~Hz}$, which has previously been identified as being in the linear regime for this system. $^{8}$

AFM images were obtained using a Bruker Multimode/ Nanoscope IIIa (Bruker, Santa Barbara, CA) atomic force microscope operating in tapping mode. The instrument was equipped with a J- Scanner (lateral scan range of $\sim 140 \mu \mathrm{m}$ ), Bruker cantilevers (model MPP-11220-10) with nominal spring constant of $40 \mathrm{~N} / \mathrm{m}$ and resonant frequency of $300 \mathrm{kHz}$, and tips with nominal tip radius of $8 \mathrm{~nm}$. Images were post-processed and analysed using the Gwyddion software package. ${ }^{27}$ Samples were prepared by pipetting a small volume of molten gel onto a mica substrate, which was then spun at $~ 1000 \mathrm{rpm}$ for 20 seconds to spread a thin layer of gel across the substrate.

FTIR measurements were performed using a Renishaw inVia Raman Microscope with a noncontact all reflective objective, attached to a Smiths IlluminatIR FTIR spectrometer. Samples were dropcast on IR reflective substrates and imaged. Baseline spectra were subtracted from all sample spectra. 
Absorbance spectra were obtained using a Cary 1E spectrophotometer. Samples were prepared by spin casting hot solutions onto UV transparent quartz at $1000 \mathrm{rpm}$.

Raman spectra were measured using a Coderg T800 triple grating spectrometer. Samples were excited at $514.5 \mathrm{~nm}$ using the output of an argon-ion laser, with scattered light collected at $90^{\circ}$ to incidence. Samples were prepared in $5 \mathrm{ml}$ glass vials, placed in the beam path for $\sim 20$ minutes prior to measurement to photo-bleach chromophores, and thus reduce the effect of fluorescence. For AFM and spectroscopy measurements, samples were left overnight at room temperature to cool and for gelation to occur. All measurements were carried out at room temperature of approximately $20^{\circ} \mathrm{C}$, well below the melting point of the gel.

\section{Results and Discussion}

To better understand the different behaviour of the two sterol-esters, computational methods were used in order to clarify the possible interaction/binding of several phytosterols (Scheme 2) to $\gamma$-oryzanol. Although commonly referred to as if it was a pure molecule, oryzanol is actually a mixture of several components, the composition of which varies depending on the strain of rice. However, the major components (see scheme 1) which account for over $85 \%$ are the 24-methylene cycloartanyl ferulate (53-57\%), cycloartenyl ferulate (27-30\%), campesteryl ferulate $(8-11 \%)$ and $\beta$-sitosteryl ferulate $(1-4 \%)$. We therefore thought it worthwhile to investigate how these different components interact with the phytosterols. 24-methylene cycloartanyl ferulate and cycloartenyl ferulate are by far the major components of $\gamma$-oryzanol, and because of their strong structural similarity with a small modification in the alkyl chain, we have used cycloartenyl ferulate for the docking simulations with the phytosterols. Cycloartenyl ferulate was also used to build the tubular structures for the MD simulations, and therefore any use of oryzanol in the simulation models is referring to cycloartenyl ferulate. 
Structural models of cycloartenyl ferulate and several phytosterols were prepared, and then docking calculations were employed to investigate their potential binding modes. All compounds exhibit docking energies from -5.00 to $-5.95 \mathrm{Kcal} / \mathrm{mol}$ (Table S1, Supporting Information). The top-ranked cluster docked conformation of sitosterol (Figure $1 \mathrm{a} / \mathrm{b}$ ) displays its sterane group stacked with the cycloartenyl ferulate's sterane group and, at the same time, a hydrogen bond between the hydroxyl group of the sitosterol and the carbonyl group of the cycloartenyl ferulate is formed. In addition, the presence of the sterane's methyl group (C30, Scheme 1) and the formed hydrogen bond prevents parallel stacking of the molecules, as can be seen in Figure 1b. Out of the top three clusters, the top-ranked cluster docked conformation of sitosterol was the highest-populated one comprising 48 out of 100 conformations, with the lowest free energy of binding estimated to be $\Delta G=-5.56 \mathrm{Kcal} / \mathrm{mol}$ (Figure $\mathrm{S} 1$ ). The next distinct cluster was quite low-populated with $\Delta G=-5.26 \mathrm{Kcal} / \mathrm{mol}$, whereas the third cluster was the second most populated with $\Delta G=-5.18 \mathrm{Kcal} / \mathrm{mol}$ (Figure S1). Importantly, the second and third cluster didn't display the hydrogen bond between the hydroxyl group of the sitosterol and the carbonyl group of the oryzanol and the conformation was antiparallel.

Regarding the docking of the other sterol compounds, as it is seen in Figure $1 \mathrm{c} / \mathrm{d}$, all the conformers with the highest docked energy exhibit a similar binding mode with that of sitosterol/cycloartenyl ferulate. Only cholestane, a molecule similar to cholesterol that lacks the -OH group and the double bond between C5 and C6 (Scheme 1c), displays a slightly different binding mode (Figure 2). This is because of the absence of the $-\mathrm{OH}$ group, and thus cholestane could not form the hydrogen bond with the carbonyl group of cycloartenyl ferulate, and only dispersion and van der Waals ( $\mathrm{vdW}$ ) interactions between the sterane cores were formed. In addition, rheological results ${ }^{8}$ have previously shown that cholestane doesn't form a gel in combination with $\gamma$-oryzanol, whereas cholesterol and sitosterol did form a gel. This small change in the chemical structure is important for the gel-forming capability of phytosterols. 
Therefore, our initial docking results suggest that all phytosterols bind to cycloartenyl ferulate in a similar manner due to their $-\mathrm{OH}$ group, which allows the formation of a wedge-shaped dimer that can in turn stack to form a helical tubule.<smiles>C/C=C(/CC[C@H](C)C1CCC2C3=CC[C@H]4C[C@@H](O)CC[C@]4(C)C3CC[C@]2(C)C1C)C(C)C(C)C</smiles>

avenasterol brassicasterol campestanol<smiles>CC(C)CCC[C@H](C)[C@H]1CCC2[C@@H]3CC=C4C[C@@H](O)CC[C@]4(C)[C@H]3CC[C@]2(C)[C@H]1C</smiles><smiles>[CH][C@@H](CCCC(C)C)[C@H]1CC[C@H]2[C@@H]3CCC4C[C@@H](O)CC[C@]4(C)[C@H]3CC[C@@]21C</smiles>

cholestanol

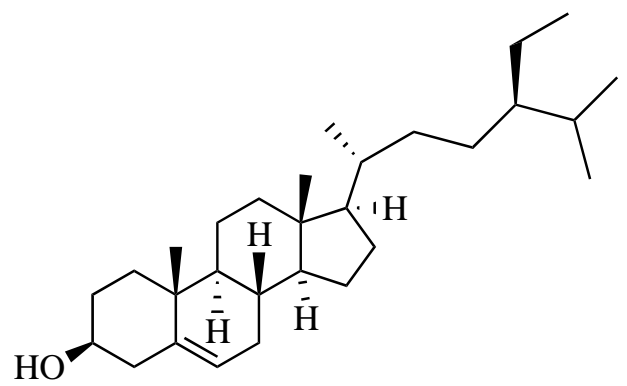

beta-sitosterol<smiles>CC(C)[C@H](C)/C=C/[C@H](C)[C@H]1CC[C@H]2C3=CC=C4C[C@@H](O)CC[C@]4(C)[C@H]3CC[C@]21C</smiles>

ergosterol

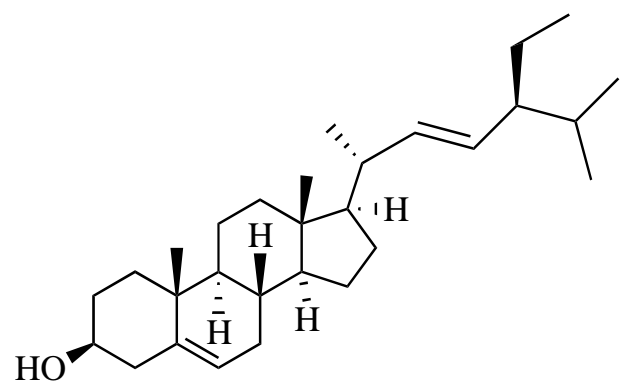

stigmasterol

Scheme 2. Chemical structures of phytosterols used in docking calculations. 
a)

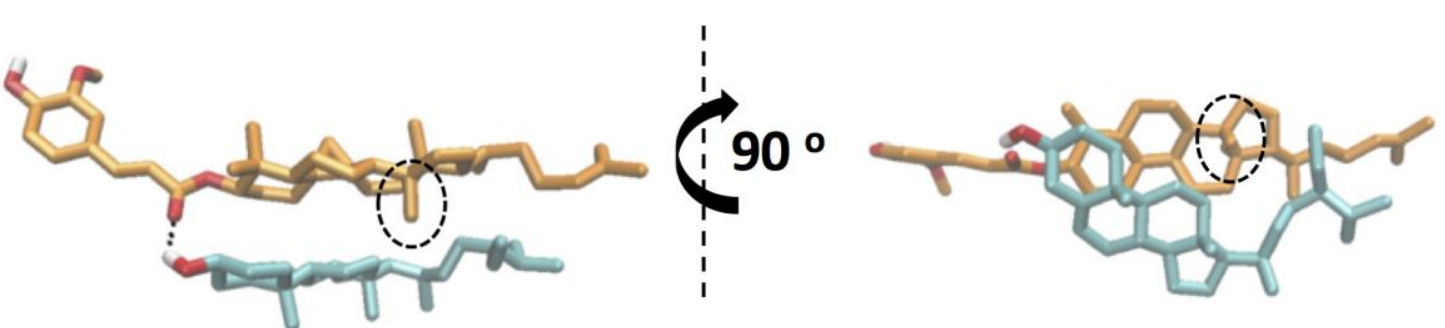

b)
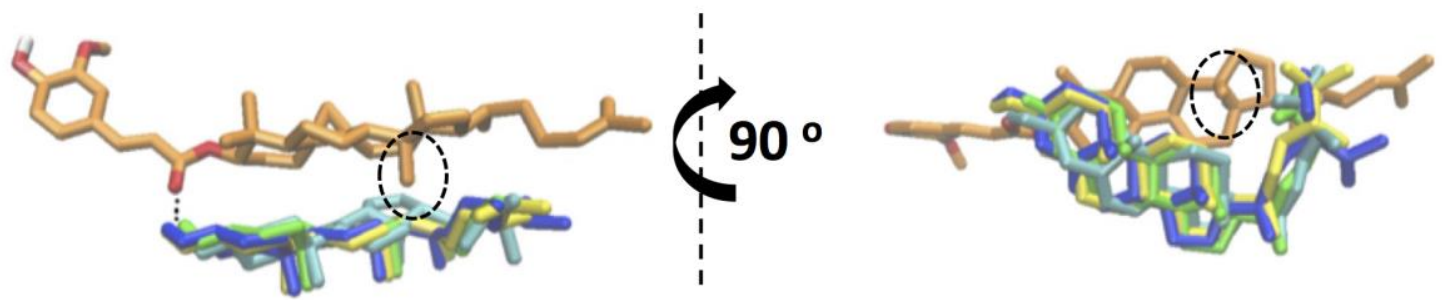

Figure 1. Views differing by $90^{\circ}$ of the lowest energy conformation for the molecular models of (a) sitosterol (cyan carbons) bound to cycloartenyl ferulate (orange carbons) and (b) phytosterols (sitosterol in cyan, cholestanol in yellow, cholesterol in green, stigmasterol in blue) bound to cycloartenyl ferulate (orange carbons). Oxygens of cycloartenyl ferulate are coloured with red and hydrogens with white. Potential hydrogen bond is indicated with dashed line, and the cycloartenyl ferulate's methyl group which prevents parallel stacking of the sterane groups is indicated with dashed circle.

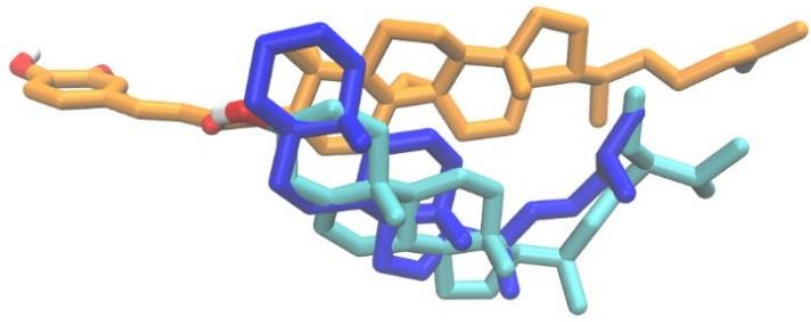

Figure 2. Molecular models of sitosterol (in cyan) and cholestane (blue) bound to cycloartenyl ferulate (orange carbons). Oxygens are coloured with red and hydrogens with white. 
In our recent study, ${ }^{17}$ we have used AFM to show that the tubules further associate into bundles, and then bifurcate and branch to form a network gel trapping the oil phase. The origin of the bundle formation is not clear yet, however it has been suggested from molecular modelling and SANS that the sterol and sterol ester form a dimer that then stacks into a tubule with the ferulate group of oryzanol sticking out from the surface. We hypothesise that fibril crosslinking arises from interactions of ferulate groups on the oryzanol on separate tubules. To test this hypothesis, we have used CHEMS in place of oryzanol where the ferulate moiety of oryzanol is replaced with a carboxylic group (Scheme 1), and we have carried out docking and MD simulations of CHEMS with sitosterol. Docking of sitosterol with CHEMS revealed four distinct conformational clusters (Figure S1, Supporting Information). None of the clusters exhibited a hydrogen bond, and only dispersion and vdW interactions between the sterane cores were observed. In regard to the top-ranked cluster, despite the fact that the two molecules had the correct orientation, they did not exhibit a hydrogen bond as the hydrogen bond angle between the $\mathrm{OH}$ group of sitosterol and the carbonyl group of CHEMS was $45^{\circ}$, which is more than the cut-off angle value of 20 degrees (Figure 3a). It has previously been shown that intermolecular hydrogen bonding between sitosterol and oryzanol is accompanied by a novel feature appearing in the FTIR spectra at $3441 \mathrm{~cm}^{-1} \cdot{ }^{7}$ In Figure $3 b$, comparing the FTIR spectra of sitosterol and oryzanol, we see a new shoulder appear at $3441 \mathrm{~cm}^{-1}$ on the low energy side of the main peak in this region, in agreement with this. However, in the system where we blend sitosterol and CHEMS no such feature appears. We take this as confirmatory experimental evidence that there is no hydrogen bonding in the sitosterol-CHEMS system, in agreement with the simulations. There are three significant ways that CHEMS differs from oryzanol - the absence of the ferulate group, differences in the acyclic tail of the steroid, and additional methyl groups hanging from the 4, 9, 10, 13 and 14 carbons of the steroidal core (Scheme 1c). Given that altering the acyclic tail of the sterols does not seem to diminish the ability of sterols to self-assemble with oryzanol, 
it suggests that the methyl groups at 4, 9,10,13 and 14 or the differences between the ferulate group and carboxylate group may be key. To test this, we performed further docking simulations between $\beta$-sitosteryl ferulate (one of the minor components of oryzanol) and sitosterol. Docking results for $\beta$-sitosteryl ferulate with sitosterol revealed a similar binding pose with the top-ranked docking conformation model of cycloartenyl ferulate and sitosterol. The top-ranked docking model displays the hydrogen bond between the hydroxyl group of the sitosterol and the carbonyl group of the sitosteryl ferulate. However, a difference in binding of sitosteryl ferulate with sitosterol is that the sterane cores are stacked almost parallel (see Figure 4a), due to the absence of the methyl groups in the sterane core at carbons C4 and C14. The top-ranked solution exhibits a mean free energy of binding $\Delta G=-6.69 \mathrm{Kcal} / \mathrm{mol}$, and the lowestenergy conformation of the top-ranked cluster displays an estimated free energy of binding $\Delta G=$ $-6.97 \mathrm{Kcal} / \mathrm{mol}$.

Analysis of the intermolecular energies after the docking simulations of the sterol esters cycloartenyl ferulate, sitosteryl ferulate and CHEMS (Table S2, Supporting Information) revealed that the major contribution to the free energy of binding was attributed to vdW and the desolvation energy. Because of the absence of the methyl groups at carbons C4 and C14, the sterane groups of sitosteryl ferulate and CHEMS were docked in parallel with the sterane group of sitosterol, which explains the lower free energies of binding in comparison with the cycloartenyl ferulate (Figure 4b). Therefore, this would suggest the key to oryzanol selfassembling with sterols into tubules, appears to be not only the ferulate group, but also the presence of methyl groups at carbons C4 and C14. Whilst the hydrogen bond doesn't contribute the most to the free energy of binding to the docked conformations (Table S2, Supporting Information), it does play an important role in the selection of a parallel conformation over an antiparallel one. Importantly, the negative values of the $\Delta G$ indicate that the formation of the dimer is a thermodynamically favoured process. This observation is in a good agreement with 
a study by Sawalha et al., ${ }^{28}$ who have used DSC data to calculate the binding energy for the sitosterol-oryzanol system. They found that the binding energy for the sitosterol-oryzanol system was approximately $\Delta G=-1 \mathrm{Kcal} / \mathrm{mol}$, which is in line with the fact that the formation of the tubules is a readily thermo-reversible process.

a)

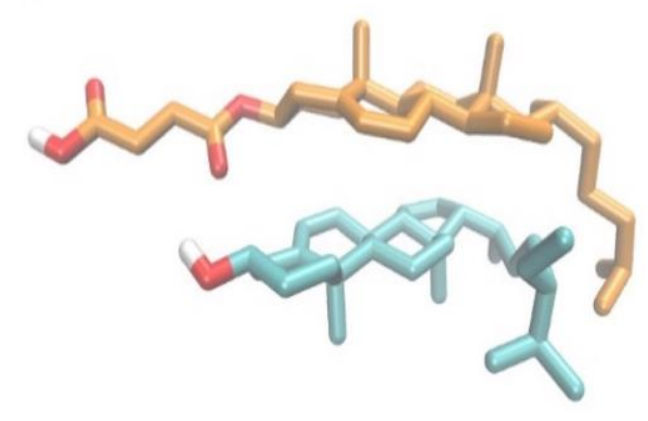

b)

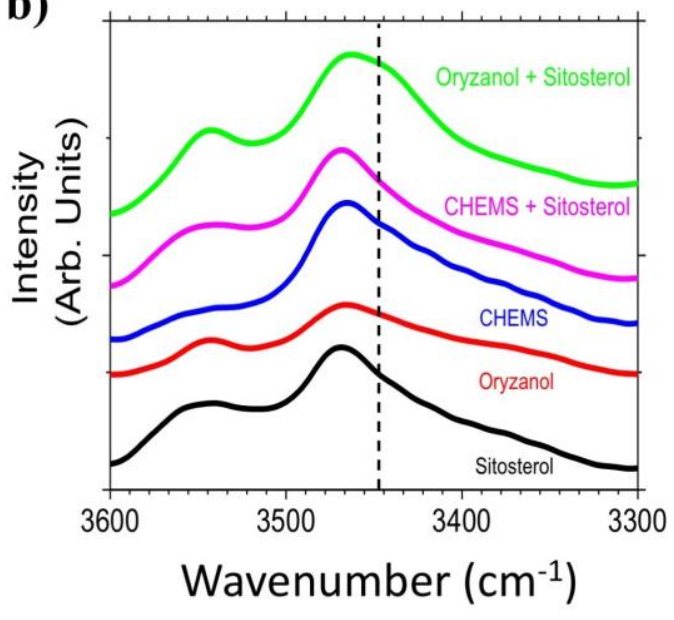

Figure 3. a) Views of the lowest energy conformation for the molecular models of sitosterol (in cyan) bound to CHEMS (orange carbons). Oxygens are coloured with red and hydrogens with white. b) FTIR spectra for sitosterol, oryzanol, CHEMS, as well as blends of sitosterol and CHEMS, and sitosterol and oryzanol.

a)

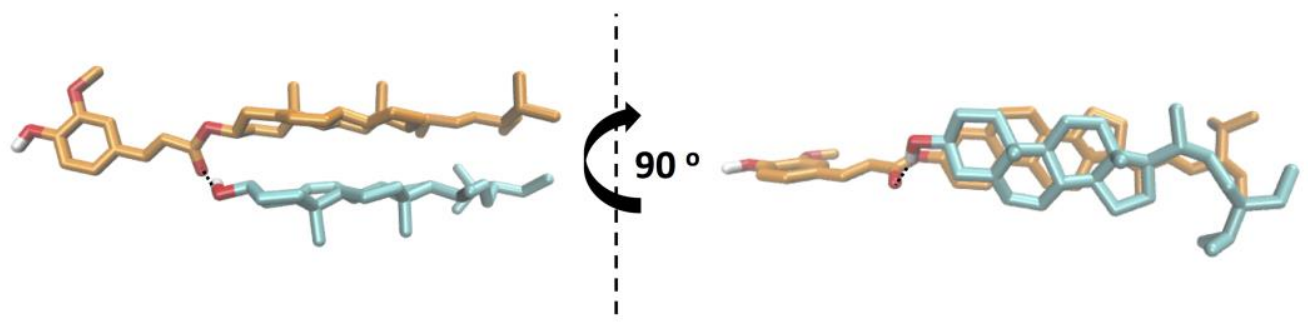

b)

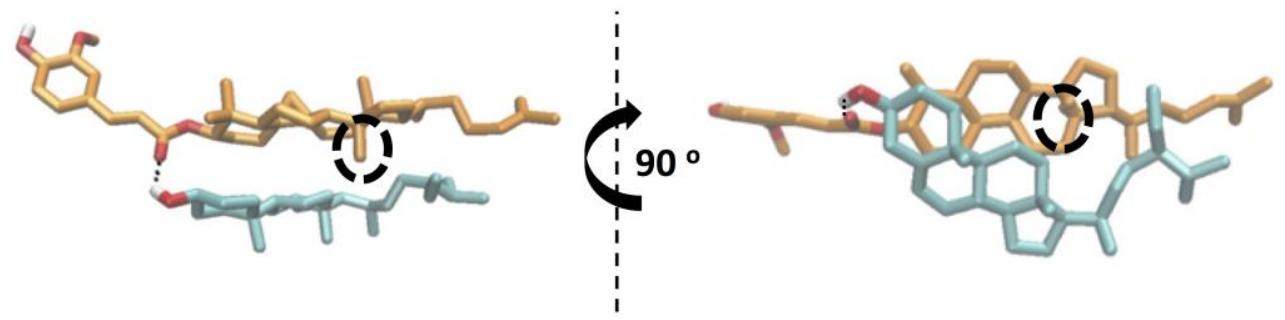

Figure 4. Views differing by $90^{\circ}$ of the lowest energy conformation for the molecular models of sitosterol (in cyan) bound to (a) sitosteryl ferulate and (b) cycloartenyl ferulate (orange carbons). Oxygens are 
coloured with red and hydrogens with white. Potential hydrogen bond is indicated with a dashed line and the cycloartenyl ferulate's methyl group which prevents parallel stacking of the sterane groups is indicated with dashed circle.
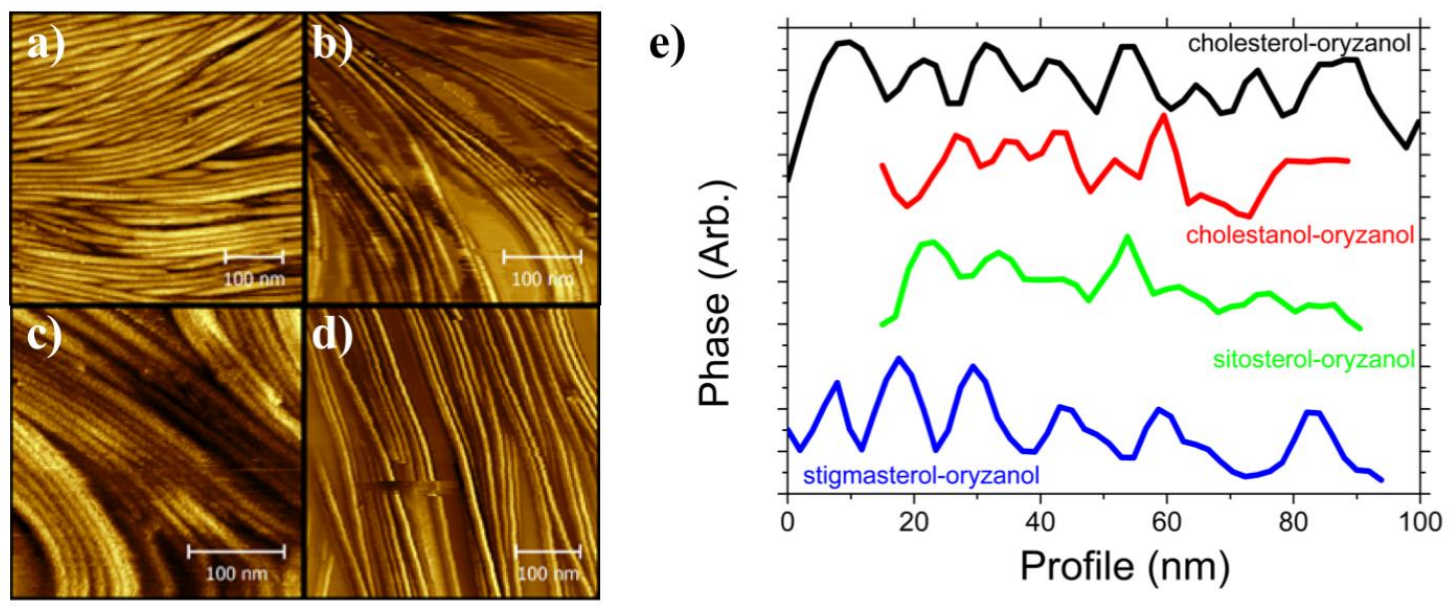

Figure 5. AFM images obtained for gels of a) cholesterol and oryzanol, b) cholestenol and oryzanol, c) sitosterol and oryzanol, and d) stigmasterol and oryzanol, spincast onto mica. e) Phase profiles from AFM images of the four sterol/sterol-ester systems.

Having used computational methods to predict dimer formation, AFM allows us to test whether tubule formation occurs as a consequence. To demonstrate tubule formation occurs in various sterol/sterol-ester systems we performed AFM on spincast samples of oryzanol blended with a range of sterols, as shown in Figure 5. For each of the four sterols blended with oryzanol, we were able to observe dense mats of $\sim 10 \mathrm{~nm}$ diameter tubules, as expected (Figure 5e). The fibrils are aligned in a parallel fashion, due to alignment with the flow field induced by spin coating. For CHEMS, however, the sample was too "wet" to be able to image with the AFM. The inability of sitosterol to form tubules and thus gel with CHEMS is further confirmed in Figure 6 where we show oscillatory rheology results for samples prepared by dispersing sterols and sterol-esters in sunflower oil. The top 4 panels are various sterols dispersed with oryzanol, whereas the bottom panel is sitosterol dispersed with CHEMS. We see that all of the sterols 
investigated quickly form very solid like gels when blended with oryzanol, but do not readily form a gel with CHEMS, as demonstrated by the fact $G^{\prime}<G^{\prime}$ ' over the timescale of the experiment. This would be somewhat surprising, as CHEMS features the steroidal core and ester bridge previously identified as the only pre-requisites of self-assembly, and has a similar solubility in sunflower oil to oryzanol. However, as demonstrated by our docking simulations and FTIR measurements, this is a consequence of the fact CHEMS and sitosterol adopt a different stacking motif from oryzanol and sitosterol.

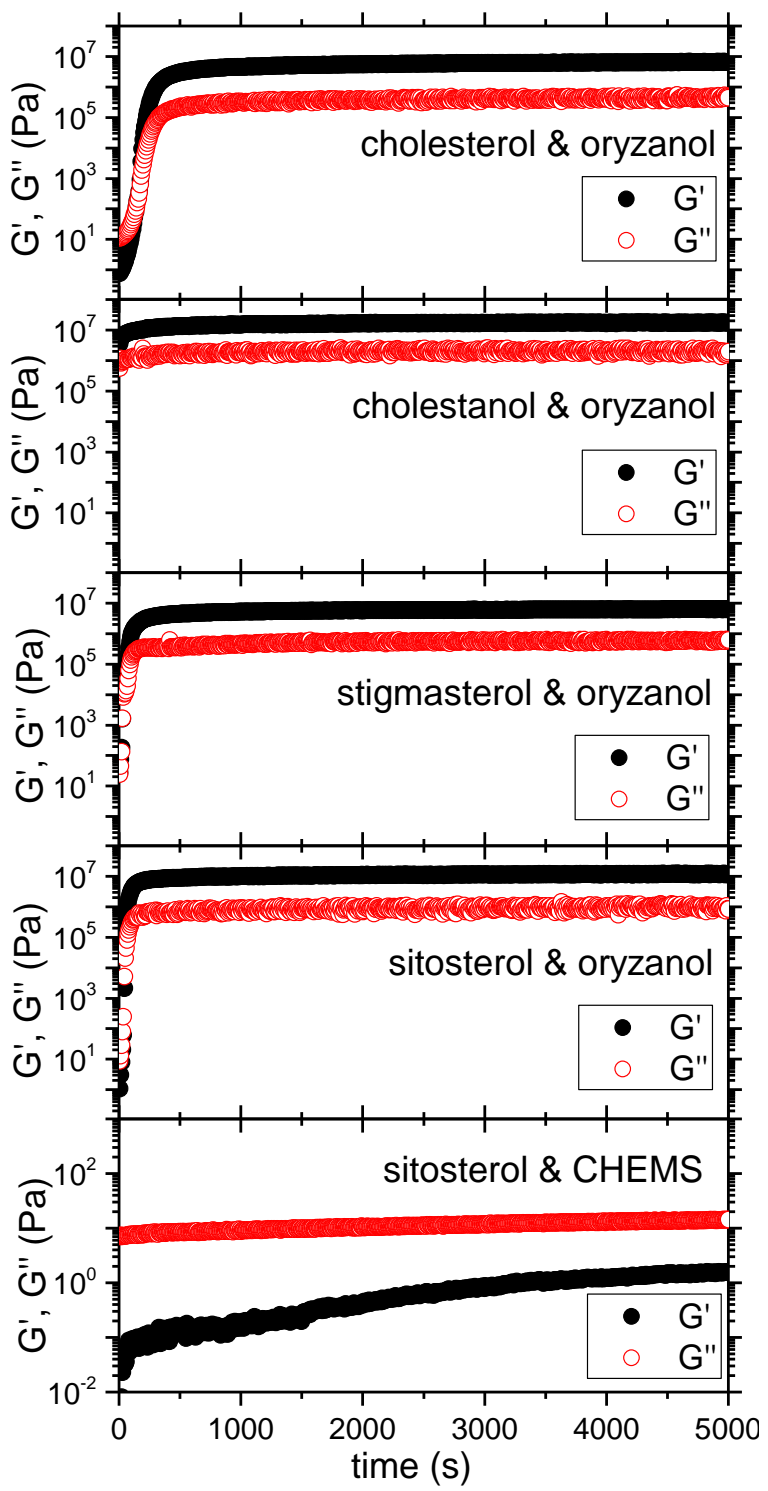

Figure 6. Oscillatory rheology results obtained immediately after pouring $10 \%$ sterol-sterol ester in sunflower oil solutions onto the bottom plate of a rheometer, and applying a $175 \mathrm{~Pa}$ stress at $10 \mathrm{~Hz}$. 
As well as docking, we employed molecular dynamics simulations of the dimers sitosteroloryzanol and sitosterol-CHEMS to examine their stability at the nanosecond timescale. The allatom MD simulations were initiated from the coordinates of the top-ranked docked models and were performed in decane for a total time of $10 \mathrm{~ns}$ for each system. The calculations were carried out using the same simulation parameters. The sitosterol-oryzanol dimer displayed remarkable stability as evidenced by the Root Mean Square Deviation (RMSD) of the corresponding $\mathrm{C}$ atoms with respect to the initial structure (Figure $\mathrm{S} 2$ ). The average value of $\approx$ $2.1 \AA$ suggests that the overall structure of the sitosterol-oryzanol dimer fluctuates around the initial coordinates at the simulation timescale. By contrast, a first indication of the replaced ferulate moiety of oryzanol with a carboxylic group is shown with the higher RMSD values of the sitosterol-CHEMS dimer, deviated significantly during the simulation time and displayed RMSD values of $\approx 7.4 \AA$. Examination of the MD trajectories of the sitosterol-oryzanol dimer revealed that the hydrogen bond between the hydroxyl group of the sitosterol and the carbonyl group of the oryzanol is remarkably stable, observed for over $91 \%$ of the simulation time (Figures S3a, Table S3). This hydrogen bond was observed for only a few ps in the system of sitosterol-CHEMS, and only hydrogen bonds between the carboxylic group of CHEMS and the hydroxyl group of sitosterol were observed (Figures S3b, Table S3). Importantly, favourable vdW contacts between the sterane groups of the dimers were observed only for the sitosteroloryzanol dimer, as for the sitosterol-CHEMS dimer the sterane groups were moved away after 1ns of simulation time (Figure S3). The MD trajectories were also used to compute the binding enthalpy of the sitosterol-oryzanol dimer. The estimated enthalpic contribution to binding for the dimer was found to be approximately $\Delta H=-2.1 \mathrm{Kcal} / \mathrm{mol}$ (Table S4), and was correlated well with the results by Sawalha et al. ${ }^{28}$ who found that the major contribution to the $\Delta G$ comes from the large negative change in the enthalpy compared with the positive entropy term, indicating that the formation of the tubules is an enthalpy-driven process. 
Consequently, we performed molecular dynamics simulations of tubules constructed using both sitosterol and oryzanol, and sitosterol and CHEMS, in a manner similar to that which we used to investigate the effect of polar solvents on tubules previously. ${ }^{12}$ The buffer of minimum $10 \AA$ between the tubule and the periodic box wall was selected so that the tubules in both systems do not interact with their own images during the simulation time (Figure S4). Whilst we have tested a cut-off $12 \AA$, however the computational cost was high and therefore a cut-off of $10 \AA$ was chosen. Snapshots of the self-assembled sitosterol-oryzanol and sitosterol-CHEMS organogel models at the beginning and after $50 \mathrm{~ns}$ of simulation time are shown in Figure 7. Visual examination of the two models revealed that the system of sitosterol-oryzanol maintained its tubular structure after the course of MDs, whereas the initial structure of sitosterol-CHEMS model underwent significant changes and the tubular structure was lost. Decane molecules interact with the sitosterol and oryzanol molecules through vdW contacts. Importantly, the use of decane as solvent for the simulations didn't affect the structure of the tubule, which is consistent with studies showing that sitosterol and oryzanol tubules can be formed in a range of organic solvents, including decane. ${ }^{21,29}$ The tubule radius of sitosteroloryzanol is stable at $\sim 50 \AA$ after $50 \mathrm{~ns}$ with only small fluctuations (Figure 8a). This value is consistent with the radius of $40-50 \AA$ (depending on the plant sterol used) obtained by scattering experiments and AFM. ${ }^{17,21}$ To quantify the structural variation of the models during the simulation time with respect to the starting conformations, we calculated the RMSD. Figure $8 \mathrm{~b}$ shows the changes of RMSD values of each carbon atom of the two models during the course of the MDs. The first nanosecond of the simulation was considered as the initial relaxation period and was ignored during the data analysis. The RMSD of the sitosterol-oryzanol model is increasing smoothly within the first $30 \mathrm{~ns}$, and then is relatively stable during the whole simulation time with no large oscillations, indicating an overall stabilization of the organogel system. The sitosterol-CHEMS organogel model produced higher RMSD values with relatively 
larger fluctuations during the whole simulation time, suggesting significant changes in the structure, which was also observed by visualizing the MD trajectories with VMD (Figure 7b). As previously outlined, several studies have shown that a hydrogen bond between the hydroxyl group of the sitosterol and the carbonyl group of the oryzanol is key to the formation of the tubular structure. ${ }^{7,8}$ During the simulation, we found that there are a lot of hydrogen bonding interactions between the hydroxyl group of the sitosterol and the carbonyl group of the oryzanol (Figure 9), as suggested in the experimental studies, enhancing the stability of the tubule. Importantly, we observed that the number of sitosterol-oryzanol hydrogen bonds was much higher than the number of sitosterol-CHEMS hydrogen bonds, as shown in Figure 8c. Specifically, the number of hydrogen bonds per dimer for the sitosterol-oryzanol system was $\approx 0.6$ and for the sitosterol-CHEMS was $\approx 0.2$. However, it is worth noting that the actual number of hydrogen bonds per dimer was higher for the sitosterol-oryzanol system, considering that during the course of the MDs some bonds may form, break and then reform several times. This is normal in MD simulations, as the violation of tight distance and angle criteria for hydrogen bonding does not necessarily mean a hydrogen bond has broken; it can be a consequence of vibrational motion of the two atoms which still remain connected by a hydrogen bond even as they move away from each other. This is consistent with our molecular docking simulations and further explains the instability of the sitosterol-CHEMS model. Furthermore, it is important to note that visual inspection of snapshots of the sitosterol-CHEMS model demonstrates that the observed hydrogen bonds were mostly randomly formed and subsequently they didn't contribute to the stability of the system. In addition, we observed that the stability of the sitosterol-oryzanol structure is further enhanced with $\pi-\pi$ contacts between the aromatic groups of the oryzanol's ferulic acid moiety. These are absent from the sitosterol-CHEMS tubules. Therefore, the sitosterol-oryzanol tubule is stabilised by a network of non-covalent cooperative interactions. 
We have previously used AFM to show that following gelation, the fibrils in the sitosteroloryzanol system do not remain isolated but tend to aggregate into bundles. To better understand the aggregation of these fibrils and how the fibrils which make up the gel structure interact in order to cause fibrils to stick together we performed MD simulations of two interacting tubules (Figure 10). Two tubule structures were placed close to each other, with the distance between them taken to be around $10 \AA$, and MD simulations were carried out for $20 \mathrm{~ns}$ under the same conditions as for the single tubule. As expected, the tubular structures of the sitosterol-CHEMS system was unstable during the course of MDs, whereas the tubular structures of the sitosteroloryzanol system was stable (as was the case for the individual tubule). Visual examination of the sitosterol-oryzanol model revealed that the aromatic groups of the ferulic acid formed vdW and $\pi-\pi$ contacts between each other. The importance of the oryzanol's ferulic acid group is shown in Figure 11, where the interfibril network of hydrogen bonds and $\pi-\pi$ contacts that is formed between the ferulic acid groups through the hydroxyl, methoxy and aromatic groups cause the fibrils to stick together. Another difference between the interactions in the interface of the two models is the higher number of interfibril hydrogen bonds in the sitosterol-oryzanol model (Figure 8d), however their contribution was limited compared to the contribution of vdW and $\pi-\pi$ contacts.

Spectroscopy gives an experimental means of probing the interactions thought to be taking place (Figure 11). UV-Vis spectroscopy shows that as the concentration of gelator is increased a new shoulder develops at $\sim 355 \mathrm{~nm}$ (Figure 12). The absorbance from $>300 \mathrm{~nm}$ is dominated by the ferulate moiety of the oryzanol, and thus the shape of this feature gives an indication of the conformation and interactions of this group. Upon increasing gelator concentration from 2 $\%$ to $10 \%$ we clearly see the growth of a new shoulder at $\sim 355 \mathrm{~nm}$. Such a shoulder is consistent with $\pi-\pi$ interactions of the aromatic ferulate group. ${ }^{30}$ Raman spectroscopy also shows that as the gel concentration is increased, there are several changes in the vibrational 
spectra. The spectrum for a $5 \%$ gel includes no features which are not also present for the two individual components. However, as the total gelator content is increased, we see changes in the spectra, most markedly at $1183 \mathrm{~cm}^{-1}$ and $1583 \mathrm{~cm}^{-1}$. These peaks are associated with modes in the ferulic acid moiety of the oryzanol, with $1183 \mathrm{~cm}^{-1}$ being assigned to the methoxy-group and $1583 \mathrm{~cm}^{-1}$ being assigned to the aromatic ring. ${ }^{31,32}$ Again, these results show that the local environment of the ferulate group is changing. As these features seem to be a consequence of increasing the gelator concentration to high values, rather than merely reaching the critical gelator concentration and forming the gel, we may logically conclude that they relate to a process other than fibril formation. This is most likely the inter-fibril aggregation we observe in MD simulations and have previously seen in AFM images of dipcast gels. ${ }^{17}$

\section{Conclusions}

A series of docking calculations in combination with molecular dynamics simulations and complementary experiments using AFM, rheology and spectroscopy provided novel insights into the interactions of several phytosterols with oryzanol. Analysis of the docked dimer models of several phytosterols with oryzanol reveal that the phytosterol's sterane group is stacked with oryzanol's sterane group and a hydrogen bond is formed between the hydroxyl group of the phytosterol and the carbonyl group of the oryzanol. In addition, we found that the presence of the cyclopentane's methyl group in oryzanol and the formed hydrogen bond prevents parallel stacking of the dimers. It is tempting to speculate that it is formation of this wedge-shaped dimer that may cause the sterols and sterol esters to form a helical tubule. On the other hand, docking simulations of sitosterol with CHEMS did not display any hydrogen bond between the two molecules, which is consistent with the FTIR spectra of sitosterol and CHEMS. The FTIR spectra of sitosterol and oryzanol in oil shows a small peak at $3441 \mathrm{~cm}^{-1}$, 
indicating intermolecular hydrogen bonding, which is in agreement with the study by den Adel et $a .^{7}$ A similar feature was not observed at $3441 \mathrm{~cm}^{-1}$ in the system of sitosterol and CHEMS, further evidence for the absence of a hydrogen bond between sitosterol and CHEMS. It seems that this lack of hydrogen bond is the reason why the sterols we have used in this study did not gel with CHEMS. This outcome was further investigated with MD simulations, where the initial self-assembled tubular configuration of sitosterol-CHEMS model underwent significant changes in the structure and the tubular structure was lost during the course of simulation time. Conversely, the initial cylindrical configuration of sitosterol and oryzanol maintained its tubular structure after the course of MDs, through a network of hydrogen bonds between the sterol's hydroxyl group and the oryzanol's carbonyl group, dispersion and vdW interactions between the sterane groups, and $\pi-\pi$ contacts between the aromatic groups of the oryzanol's ferulic acid moiety. Importantly, by using MD simulations we have identified a possible mechanism for how two tubules of sitosterol with oryzanol stick together to form bundles. We found that an interfibril network primarily of $\mathrm{vdW}$ interactions, and $\pi-\pi$ contacts (with some likely role for hydrogen bonds and dispersion interactions) that form between the ferulic acid groups of oryzanol through the aromatic groups as well as the hydroxyl, methoxy groups cause the fibrils to stick together. 

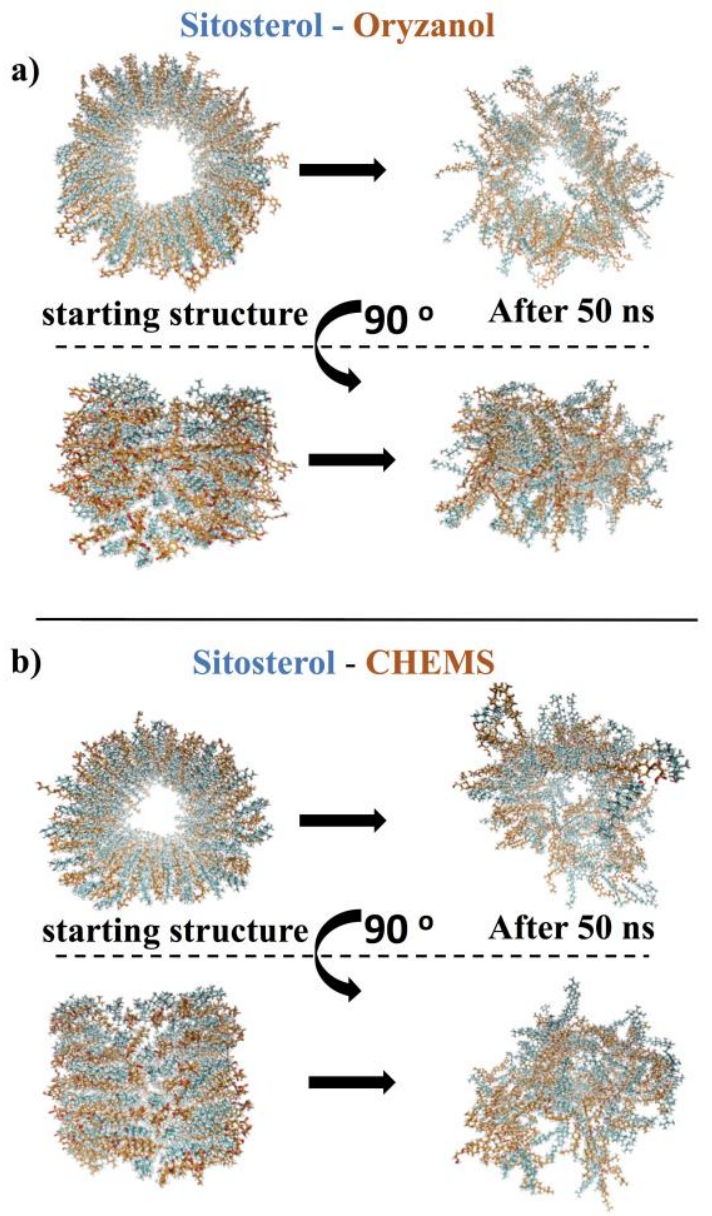

Figure 7. Snapshots of the (a) sitosterol-oryzanol and (b) CHEMS-oryzanol organogels at the beginning and at the end of the course of the MD simulation. Sitosterol is shown with cyan carbons and oryzanol and CHEMS with orange carbons. 
a)

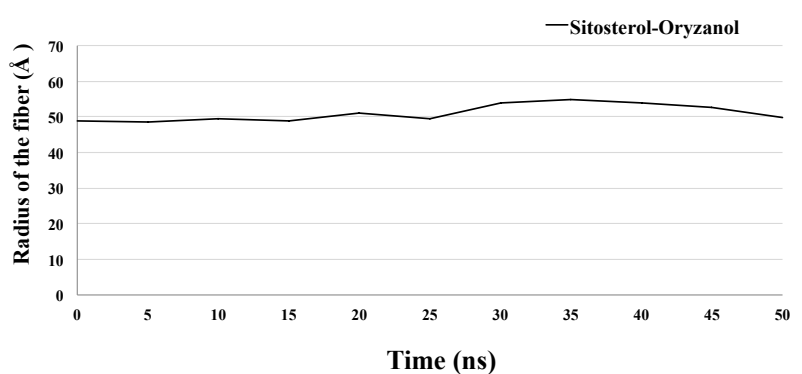

b)

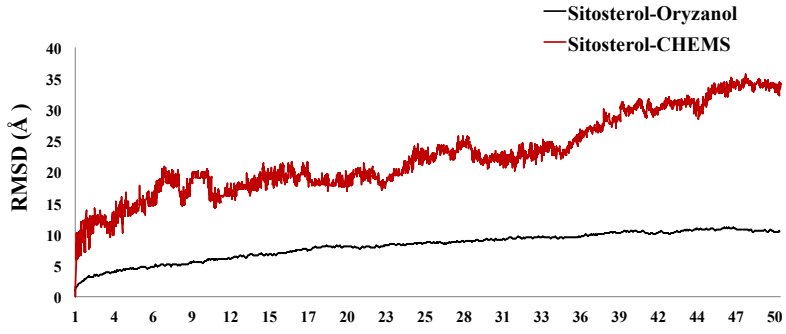

Time (ns)

c)

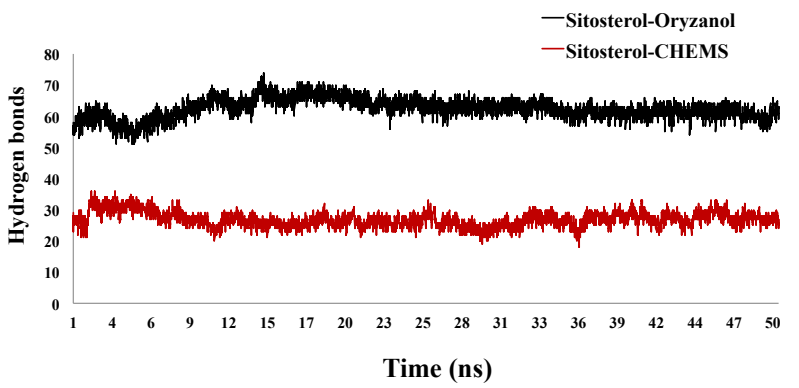

d)

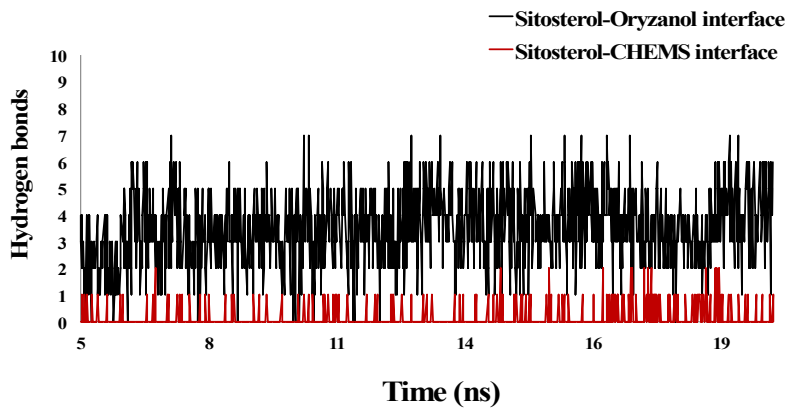

Figure 8. a) Radius of the sitosterol-oryzanol tubular model as a function of simulation time, b) Timedependent RMSD from the starting conformations, $c$ ) Number of hydrogen bonds between the hydroxyl group of the sitosterol and the carbonyl group of the oryzanol/CHEMS, d) Number of hydrogen bonds in the interfaces of the two tubules (the two tubules started to interact after $5 \mathrm{~ns}$ of simulation time). Alignments and measurements were performed for the carbon atoms. 


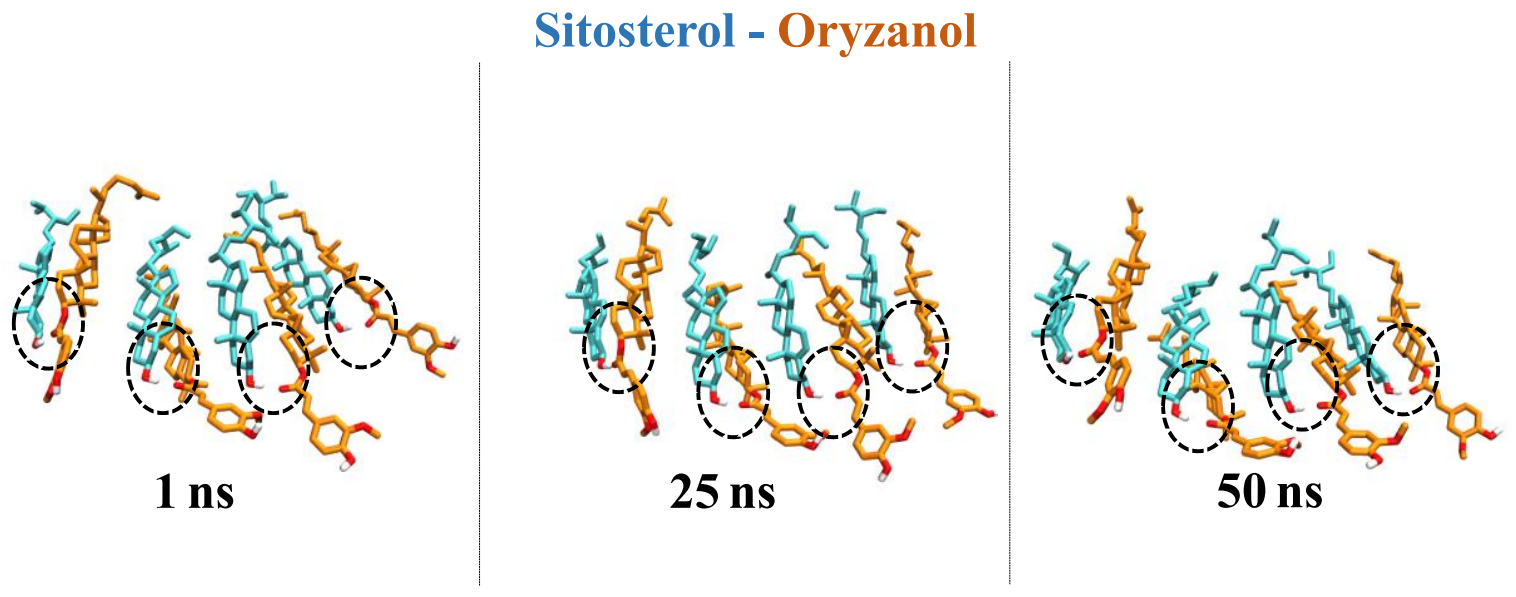

Figure 9. Snapshots of four consecutive sitosterol-oryzanol pairs during the MD simulation. The other sitosterol-oryzanol pairs of the tubule were omitted for visual clarity. Intermolecular hydrogen bonds between the hydroxyl group of the sitosterol and the carbonyl group of the oryzanol are outlined with black rings.
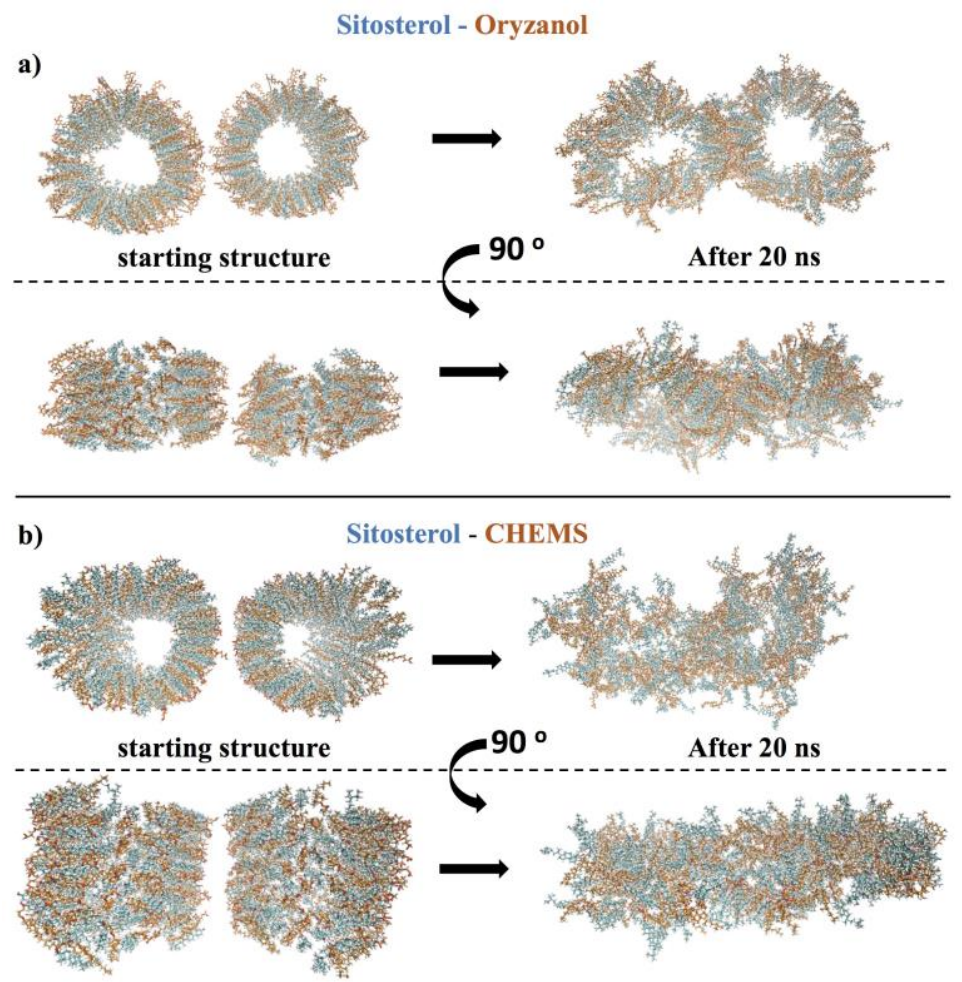

Figure 10. Snapshots of two tubules of (a) sitosterol-oryzanol and (b) sitosterol-CHEMS at the beginning and at the end of the course of the MD simulation. Sitosterol is shown with cyan carbons and oryzanol and CHEMS with orange carbons. 


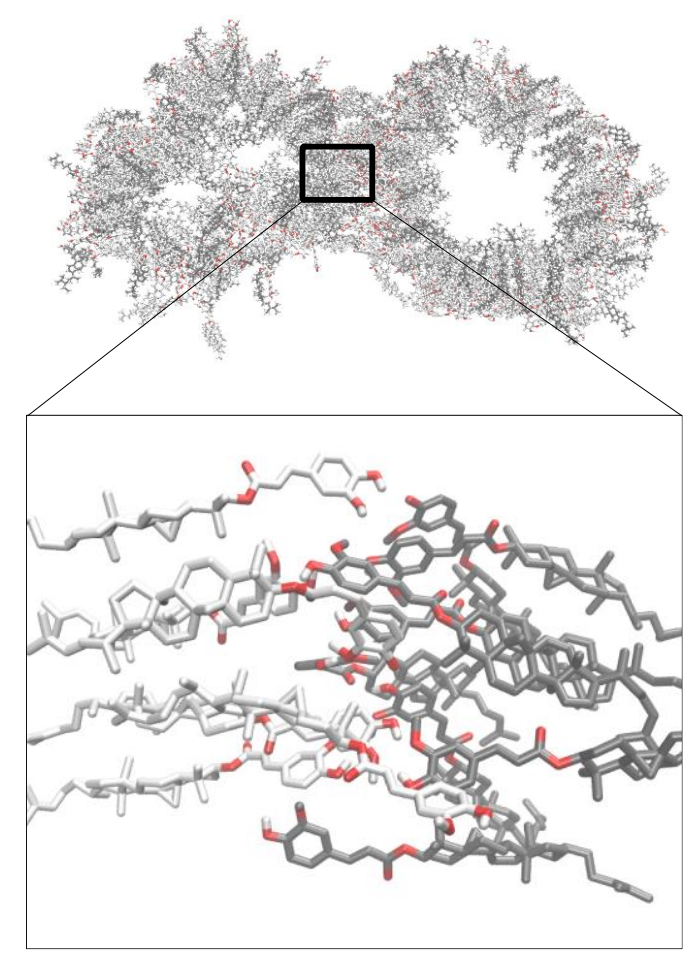

Figure 11. Hydrogen bonds, $v d W$ interactions and $\pi-\pi$ contacts between the ferulic acid groups of oryzanol in the interface of two tubules.

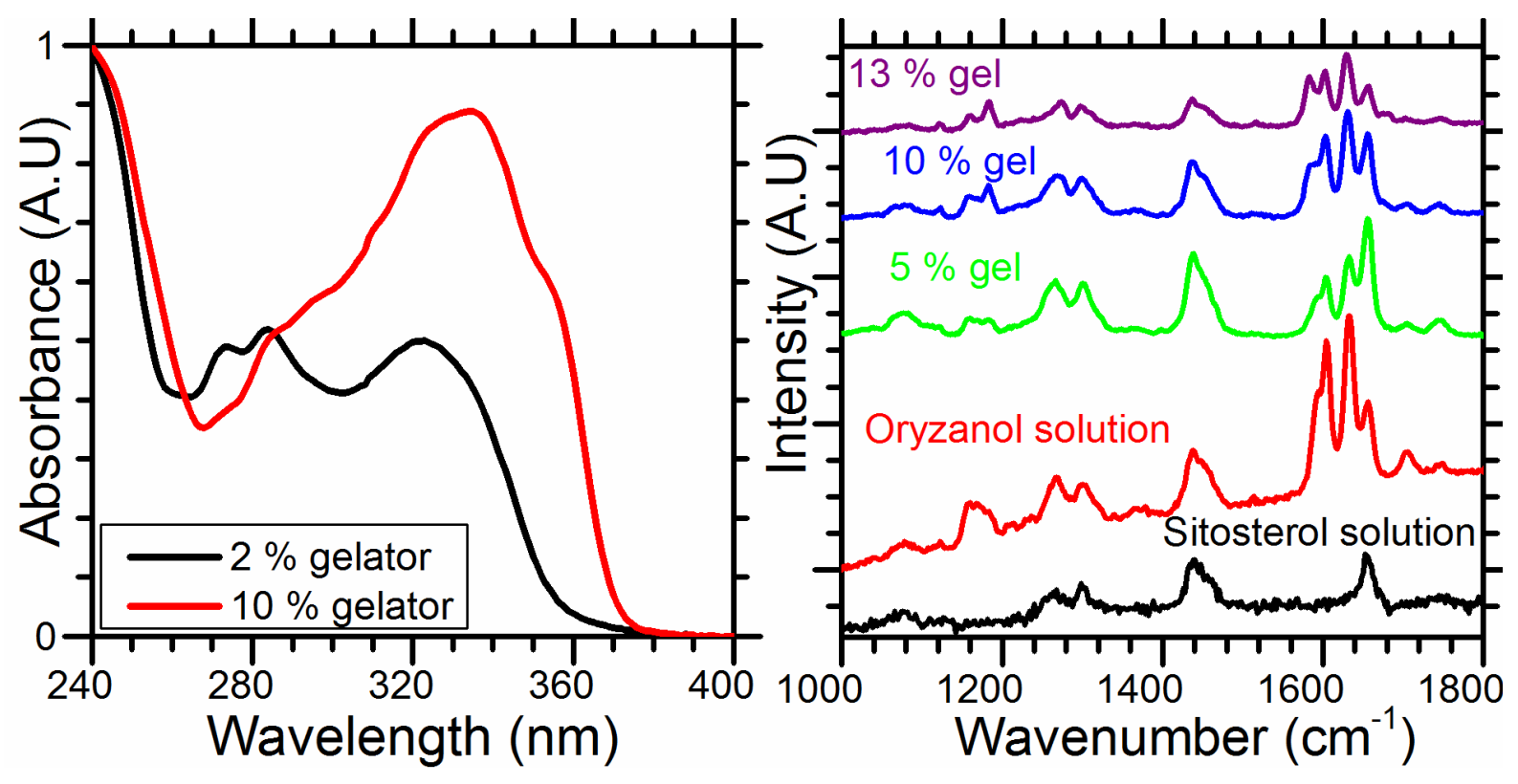

Figure 12. Normalised UV-visible absorbance spectra of $2 \%$ and $10 \%$ sitosterol-oryzanol in sunflower oil samples (left), and Raman spectroscopy results for various concentrations of sitosterol and oryzanol in sunflower oil, as well as individual materials in sunflower oil (right). 


\section{Supporting Information}

Further details of the simulation protocols are given in the supporting information. Results of the docking calculations for the top conformational clusters of phytosterols docked to $\gamma$ oryzanol are shown in Table S1. Table S2 shows the final intermolecular energies after docking simulations of the sterol esters with sitosterol, and Table S3 shows the hydrogen bonds of the sterol esters with sitosterol dimers during the simulation time. Table S4 shows the computed binding enthalpy of the sitosterol-oryzanol dimer. Histogram of the free energy of binding for the clusters of the sitosterol-oryzanol and sitosterol-CHEMS systems is shown in Figure S1. Snapshots of the sitosterol-oryzanol and sitosterol-CHEMS dimers during the simulation time and the time-dependent RMSD are shown in figure S2 and S3, respectively. Figure S4 shows snapshots of the sitosterol-oryzanol system in the primary cell together with two replicas.

\section{Acknowledgements}

This work was supported by the BBSRC DRINC project. [BBSRC Grants: BB/M027449/1 (HWU) and BB/M027597/1 (UoE)]. This project made use of time on ARCHER granted via the UK High-End Computing Consortium for Biomolecular Simulation, HECBioSim (http://hecbiosim.ac.uk), supported by EPSRC (grant no. EP/L000253/1). GOL thanks the Royal Society of Edinburgh/Scottish Government for a personal fellowship.

\section{References}

1. Marangoni, A. G.; Garti, N., An Overview of the Past, Present, and Future of Organogels. In Edible Oleogels, Elsevier: 2011; pp 1-17.

2. Katan, M. B.; Grundy, S. M.; Jones, P.; Law, M.; Miettinen, T.; Paoletti, R.; Stresa Workshop, P., Efficacy and safety of plant stanols and sterols in the management of blood cholesterol levels. Mayo Clin Proc 2003, 78, (8), 965-78. 
3. Xu, Z.; Hua, N.; Godber, J. S., Antioxidant activity of tocopherols, tocotrienols, and gamma-oryzanol components from rice bran against cholesterol oxidation accelerated by 2,2'azobis(2-methylpropionamidine) dihydrochloride. J Agric Food Chem 2001, 49, (4), 2077-81. 4. Rogers, E. J.; Rice, S. M.; Nicolosi, R. J.; Carpenter, D. R.; McClelland, C. A.; Romanczyk, L. J., Identification and quantitation of $\gamma$-oryzanol components and simultaneous assessment of tocols in rice bran oil. Journal of the American Oil Chemists' Society 1993, 70, (3), 301-307.

5. $\quad$ Bot, A.; den Adel, R.; Roijers, E. C.; Regkos, C., Effect of Sterol Type on Structure of Tubules in Sterol $+\gamma$-Oryzanol-Based Organogels. Food Biophysics 2009, 4, 266-272.

6. Pernetti, M.; van Malssen, K. F.; Flöter, E.; Bot, A., Structuring of edible oils by alternatives to crystalline fat. Current Opinion in Colloid \& Interface Science 2007, 12, 221231.

7. den Adel, R.; Heussen, P. C. M.; Bot, A., Effect of water on self-assembled tubules in $\beta$-sitosterol $+\gamma$-oryzanol-based organogels. Journal of Physics: Conference Series 2010, 247 , 012025 .

8. Bot, A.; Agterof, W. G. M., Structuring of edible oils by mixtures of $\gamma$-oryzanol with $\beta$-sitosterol or related phytosterols. Journal of the American Oil Chemists' Society 2006, 83, 513-521.

9. Tekin, E. D., Molecular dynamics simulations of self-assembled peptide amphiphile based cylindrical nanofibers. RSC Adv. 2015, 5, (82), 66582-66590.

10. Sun, Y.; Qian, Z.; Guo, C.; Wei, G., Amphiphilic Peptides A6K and V6K Display Distinct Oligomeric Structures and Self-Assembly Dynamics: A Combined All-Atom and Coarse-Grained Simulation Study. Biomacromolecules 2015, 16, (9), 2940-9.

11. Jones, C. D.; Kennedy, S. R.; Walker, M.; Yufit, D. S.; Steed, J. W., Scrolling of Supramolecular Lamellae in the Hierarchical Self-Assembly of Fibrous Gels. Chem 2017, 3, (4), 603-628.

12. Matheson, A.; Dalkas, G.; Mears, R.; Euston, S. R.; Clegg, P. S., Stable emulsions of droplets in a solid edible organogel matrix. Soft Matter 2018, 14, (11), 2044-2051.

13. Matheson, A. B.; Dalkas, G.; Gromov, A.; Euston, S. R.; Clegg, P. S., The development of phytosterol-lecithin mixed micelles and organogels. Food Funct 2017, 8, (12), 4547-4554.

14. Lee, O.-S.; Stupp, S. I.; Schatz, G. C., Atomistic Molecular Dynamics Simulations of Peptide Amphiphile Self-Assembly into Cylindrical Nanofibers. Journal of the American Chemical Society 2011, 133, (10), 3677-3683.

15. Sasselli, I. R.; Pappas, C. G.; Matthews, E.; Wang, T.; Hunt, N. T.; Ulijn, R. V.; Tuttle, T., Using experimental and computational energy equilibration to understand hierarchical selfassembly of Fmoc-dipeptide amphiphiles. Soft Matter 2016, 12, (40), 8307-8315.

16. Morris, G. M.; Huey, R.; Lindstrom, W.; Sanner, M. F.; Belew, R. K.; Goodsell, D. S.; Olson, A. J., AutoDock4 and AutoDockTools4: Automated docking with selective receptor flexibility. J Comput Chem 2009, 30, (16), 2785-91.

17. Matheson, A. B.; Koutsos, V.; Dalkas, G.; Euston, S.; Clegg, P., Microstructure of betaSitosterol:gamma-Oryzanol Edible Organogels. Langmuir 2017, 33, (18), 4537-4542.

18. Bot, A.; Flöter, E., Edible Oil Organogels Based on Self-assembled $\beta$-sitosterol $+\gamma$ oryzanol Tubules. In Edible Oleogels, Second Edi ed.; Elsevier: 2011; pp 49-79.

19. Hawkins, P. C.; Skillman, A. G.; Warren, G. L.; Ellingson, B. A.; Stahl, M. T., Conformer generation with OMEGA: algorithm and validation using high quality structures from the Protein Databank and Cambridge Structural Database. J Chem Inf Model 2010, 50, (4), 572-84.

20. Bot, A.; den Adel, R.; Roijers, E. C., Fibrils of $\gamma$-Oryzanol $+\beta$-Sitosterol in Edible Oil Organogels. Journal of the American Oil Chemists' Society 2008, 85, 1127-1134. 
21. Bot, A.; Gilbert, E. P.; Bouwman, W. G.; Sawalha, H.; den Adel, R.; Garamus, V. M.; Venema, P.; van der Linden, E.; Flöter, E., Elucidation of density profile of self-assembled sitosterol + oryzanol tubules with small-angle neutron scattering. Faraday Discussions 2012, $158,223$.

22. Case, D. A.; Cerutti, D. S.; Cheatham, T. E.; Darden, T. A.; Duke, R. E.; Giese, T. J.; Gohlke, H.; Goetz, A. W.; Greene, D.; Homeyer, N.; Izadi, S.; Kovalenko, A.; Lee, T. S.; LeGrand, S.; Li, P.; Lin, C.; Liu, J.; Luchko, T.; Luo, R.; Mermelstein, D.; Merz, K. M.; Monard, G.; Nguyen, H.; Omelyan, I.; Onufriev, A.; Pan, F.; Qi, R.; Roe, D. R.; Roitberg, A.; Sagui, C.; Simmerling, C. L.; Botello-Smith, W. M.; Swails, J.; Walker, R. C.; Wang, J.; Wolf, R. M.; Wu, X.; Xiao, L.; York, D. M.; Kollman, P. A., Amber 2016. University of California, San Francisco 2016.

23. Wang, J.; Wolf, R. M.; Caldwell, J. W.; Kollman, P. A.; Case, D. A., Development and testing of a general amber force field. J Comput Chem 2004, 25, (9), 1157-74.

24. Hornak, V.; Abel, R.; Okur, A.; Strockbine, B.; Roitberg, A.; Simmerling, C., Comparison of multiple Amber force fields and development of improved protein backbone parameters. Proteins 2006, 65, (3), 712-25.

25. Roe, D. R.; Cheatham, T. E., 3rd, PTRAJ and CPPTRAJ: Software for Processing and Analysis of Molecular Dynamics Trajectory Data. J Chem Theory Comput 2013, 9, (7), 308495.

26. Humphrey, W.; Dalke, A.; Schulten, K., VMD: visual molecular dynamics. $J$ Mol Graph 1996, 14, (1), 33-8, 27-8.

27. Nečas, D.; Klapetek, P., Gwyddion: an open-source software for SPM data analysis. Central European Journal of Physics 2012, 10, (1), 181-188.

28. Sawalha, H.; Venema, P.; Bot, A.; Flöter, E.; van der Linden, E., The Influence of Concentration and Temperature on the Formation of ??-Oryzanol + ??-Sitosterol Tubules in Edible Oil Organogels. Food Biophysics 2011, 6, 20-25.

29. Sawalha, H.; den Adel, R.; Venema, P.; Bot, A.; Floter, E.; van der Linden, E., Organogel-Emulsions with Mixtures of beta-Sitosterol and gamma-Oryzanol: Influence of Water Activity and Type of Oil Phase on Gelling Capability. Journal of Agricultural and Food Chemistry 2012, 60, 3462-3470.

30. Pan, G. X.; Thomson, C. I.; Leary, G. J., Uv-Vis. Spectroscopic Characteristics of Ferulic Acid and Related Compounds. Journal of Wood Chemistry and Technology 2002, 22, (2-3), 137-146.

31. Mandak, E.; Zhu, D.; Godany, T. A.; Nyström, L., Fourier Transform Infrared Spectroscopy and Raman Spectroscopy as Tools for Identification of Steryl Ferulates. Journal of Agricultural and Food Chemistry 2013, 61, (10), 2446-2452.

32. Sebastian, S.; Sundaraganesan, N.; Manoharan, S., Molecular structure, spectroscopic studies and first-order molecular hyperpolarizabilities of ferulic acid by density functional study. Spectrochimica Acta Part A: Molecular and Biomolecular Spectroscopy 2009, 74, (2), 312-323. 
Table of Contents

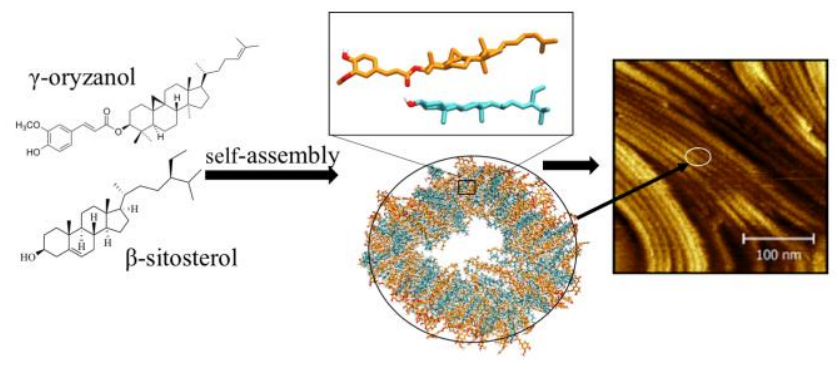

For Table of Contents Only 


\title{
Supporting Information
}

\section{Molecular interactions behind the self-assembly and microstructure of mixed sterol organogels}

\author{
Georgios Dalkas*1, Andrew B. Matheson², Hugh Vass², Andrei Gromov³, Gareth 0 . \\ Lloyd $^{4}$, Vasileios Koutsos ${ }^{5}$, Paul S. Clegg ${ }^{2}$, Stephen R. Euston ${ }^{1}$
}

1- School of Engineering and Physical Sciences, Institute of Mechanical Process and Energy Engineering, John Muir Building, Heriot-Watt University, Edinburgh

2- School of Physics and Astronomy, University of Edinburgh, James Clerk Maxwell Building, Edinburgh

3- EaStChem, School of Chemistry, University of Edinburgh, Edinburgh

4- School of Engineering and Physical Sciences, Institute of Chemical Sciences, William Perkin Building, Heriot-Watt University, Edinburgh

5- School of Engineering, Institute for Materials and Processes, University of Edinburgh, Sanderson Building, Edinburgh

* Corresponding author: G.Dalkas@hw.ac.uk

\section{Contents:}

$1 \quad$ Further details of the simulation protocols $\quad$ S2

$2 \quad$ Table S1. Docking calculations of phytosterols docked to $\gamma$-oryzanol S4

3 Table S2. Intermolecular energies of the sterol esters docked to $\quad$ S4 sitosterol

$4 \quad$ Table S3. Hydrogen bonds of the sterol esters - sitosterol dimers $\quad$ S4

5 Table S4. Computed binding enthalpy of the sitosterol-oryzanol dimer S5

6 Figure S1. Histogram of the free energy of binding for the clusters of S6 the sitosterol-oryzanol and sitosterol-CHEMS systems

$7 \quad$ Figure S2. Time-dependent RMSD from the starting conformations $\quad$ S7

8 Figure S3. Snapshots of the sitosterol-oryzanol and sitosterol-CHEMS S7

9 Figure S4. Snapshots of the sitosterol-oryzanol system in the primary S8

10 References $\quad$ S9 


\section{Simulation protocols}

Force field parameterization. The AMBER v16 package was used for simulation of the systems. Force-field parameters for all the compounds were prepared using the Antechamber package ${ }^{1}$, and the AM1-BCC atomic charges. The ff14SB ${ }^{2}$ force field parameters and the General-AMBER force field (GAFF) ${ }^{1}$ were applied to the structure atoms using the XLEaP module of AMBER16.

Molecular dynamics equilibration. A three-stage equilibration protocol, consisting of energy minimization and MD simulations, was used to eliminate any unfavourable interactions which may have occurred as a result of model building process and to gently adjust the starting structure to the molecular mechanics force field prior to production of MD simulations. The calculations for all the systems (dimers, tubules and two tubules) were carried out using the same simulation parameters. The energy of the initial structure was first minimized using 5000 steps of steepest descent minimization followed by 5000 steps of conjugate gradient minimization. Next, the system was slowly heated to the production temperature of $300 \mathrm{~K}$ in two stages. All atoms in the system were restrained to their initial coordinates during the heating in order to prevent large structural deviations, using a harmonic restraint force constant of 10 $\mathrm{kcal} \mathrm{mol}^{-1} \AA^{-2}$. In the first heating stage the system target temperature was slowly raised from 0 to $100 \mathrm{~K}$ for 10 ps with a constant volume simulation using the Langevin thermostat with a collision frequency of $1.0 \mathrm{ps}^{-1} \cdot{ }^{3}$ The second phase of heating slowly increased the temperature to the production temperature of $300 \mathrm{~K}$ over 90 ps with constant pressure periodic boundary conditions. In the final MD equilibration step of $100 \mathrm{ps}$, no atomic coordinates were constrained.

Molecular dynamics production runs. After heating the system, a production run of $50 \mathrm{~ns}$ of molecular dynamics was simulated at constant temperature and pressure using the Langevin thermostat at $300 \mathrm{~K}$ with a collision frequency of $1.0 \mathrm{ps}^{-1}$. The pressure was regulated at $1 \mathrm{bar}$ 
using the Berendsen weak-coupling algorithm with a relaxation time of $2.0 \mathrm{ps} .{ }^{4}$ The SHAKE algorithm ${ }^{5}$ was used to constrain bonds involving hydrogen with a relative tolerance of $1 \times 10^{-7}$. The Particle Mesh Ewald (PME) method was used for long-range electrostatic and van der Waals interactions, ${ }^{6}$ with a real-space cut-off of $8 \AA$. A time step of 1 fs was used for equilibration and increased to $2 \mathrm{fs}$ for the production of MD.

Calculation of Mean Potential Energies and Binding Enthalpy of the sitosterol-oryzanol dimer.

We used the SANDER module of AMBER 16 to compute the potential energy for each trajectory snapshot when all the atoms are present (total), when only the solvent is present (solvent-solvent), and when only the solute is present (solute-solute). The binding enthalpy was computed as follows:

$\Delta \mathrm{H}=\langle\mathrm{H}\rangle_{\text {complex }}+\langle\mathrm{H}\rangle_{\text {solvent }^{-}}\langle\mathrm{H}\rangle_{\text {oryzanol- }^{-}}\langle\mathrm{H}\rangle_{\text {sitosterol }}$

where $\langle\mathrm{H}\rangle_{\text {complex }},\langle\mathrm{H}\rangle_{\text {decane, }}\langle\mathrm{H}\rangle_{\text {oryzanol, }}$ and $\langle\mathrm{H}\rangle_{\text {sitosterol }}$ are the Boltzmann averaged total potential energies for the sitosterol-oryzanol dimer, solvent (decane), oryzanol, and sitosterol simulations, respectively. Additional details can be found in the study by Fenley et al.. ${ }^{7}$ 


\section{Tables}

Table S1. Results of the docking calculations for the top conformational clusters of phytosterols docked to $\gamma$-oryzanol. The results are ranked by the lowest free energy of binding $\Delta G$ for each conformational cluster.

The estimated mean free energy of binding $\Delta G$ is the mean value of each conformational cluster that was clustered with a RMSD cut-off of $2.0 \AA$ and its population is given as percent.

\begin{tabular}{ccccc}
\hline Phytosterol & $\begin{array}{c}\text { Cluster } \\
\text { Rank }\end{array}$ & $\begin{array}{c}\text { Population } \\
(\boldsymbol{\%})\end{array}$ & $\begin{array}{c}\text { Mean } \boldsymbol{\Delta G} \\
\left(\text { Kcal·mol }^{-1}\right)\end{array}$ & $\begin{array}{c}\text { Lowest } \Delta \boldsymbol{G} \\
\left(\text { Kcal-mol }^{\mathbf{- 1}}\right)\end{array}$ \\
\hline $\boldsymbol{\Delta 7 - A v e n a s t o l ~}$ & 1 & 63 & -5.40 & -5.95 \\
\hline Brassicasterol & 1 & 28 & -5.65 & -5.84 \\
\hline Campestanol & 1 & 44 & -5.32 & -5.66 \\
\hline Campesterol & 1 & 33 & -5.33 & -5.66 \\
\hline 5a-Cholestane & 1 & 42 & -5.46 & -5.74 \\
\hline Cholesterol & 1 & 52 & -5.15 & -5.50 \\
\hline Cholestanol & 1 & 63 & -5.47 & -5.83 \\
\hline Ergosterol & 1 & 48 & -5.59 & -5.82 \\
\hline Sitosterol & 1 & 48 & -5.09 & -5.56 \\
\hline Stigmasterol & 1 & 23 & -5.44 & -5.73 \\
\hline
\end{tabular}

Table S2. The final intermolecular energies after docking simulations of the sterol esters with sitosterol.

\begin{tabular}{|c|c|c|c|c|c|c|c|}
\hline Sterol ester & Sterol & $\begin{array}{l}\text { Lowest } \Delta G \\
\left({\left.\text { Kcal } \cdot \mathrm{mol}^{-1}\right)}^{-1}\right)\end{array}$ & $\begin{array}{c}\text { Electrostatic } \\
\text { energy } \\
\left({\left.\mathrm{Kcal} \cdot \mathrm{mol}^{-1}\right)}^{-1}\right)\end{array}$ & $\begin{array}{c}\text { Hbond } \\
\left({\left.\text { Kcal } \cdot \text { mol }^{-1}\right)}^{-1}\right)\end{array}$ & $\begin{array}{c}\text { vdW } \\
\left(\text { Kcal·mol }^{-1}\right)\end{array}$ & $\begin{array}{l}\text { Desolvation } \\
\text { energy } \\
\left(\text { Kcal·mol }^{-1}\right)\end{array}$ & 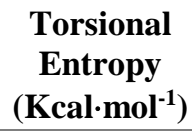 \\
\hline $\begin{array}{l}\text { cycloartenyl } \\
\text { ferulate }\end{array}$ & Sitosterol & -5.56 & -0.04 & -0.56 & -5.52 & -1.52 & 2.09 \\
\hline $\begin{array}{l}\text { sitosteryl } \\
\text { ferulate }\end{array}$ & Sitosterol & -6.97 & -0.04 & -0.42 & -6.78 & -1.86 & 2.09 \\
\hline CHEMS & Sitosterol & -6.47 & -0.08 & -0.40 & -6.36 & -1.72 & 2.09 \\
\hline
\end{tabular}

Table S3. Hydrogen bonds of the sterol esters with sitosterol dimers during the simulation time.

\begin{tabular}{|c|c|c|c|c|c|}
\hline Acceptor & $\begin{array}{l}\text { Hydrogen } \\
\text { Donor }\end{array}$ & Time (ns) & $\begin{array}{c}\text { Percent of } \\
\text { simulation } \\
\text { time }(\%)\end{array}$ & $\begin{array}{c}\text { Average } \\
\text { distance }(\AA)\end{array}$ & $\begin{array}{c}\text { Average } \\
\text { angle }\end{array}$ \\
\hline Oryzanol@O2a & Sitosterol@H1 $^{b}$ & 9.17 & 91.7 & 2.84 & 160.91 \\
\hline 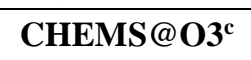 & Sitosterol@H1 & 4.59 & 45.9 & 2.88 & 156.87 \\
\hline${\mathrm{CHEMS} @ O 4^{\mathrm{d}}}$ & Sitosterol@H1 & 4.12 & 41.2 & 2.82 & 157.05 \\
\hline CHEMS@O2 & Sitosterol@H1 & 0.04 & 0.42 & 2.79 & 145.31 \\
\hline
\end{tabular}

${ }^{a}$ Oxygen of the carbonyl group of oryzanol, ${ }^{b}$ hydrogen of the hydroxyl group of sitosterol, ${ }^{\mathrm{c}, \mathrm{d}}$ oxygens of the carboxylic group of CHEMS, ${ }^{\mathrm{e}}$ oxygen of the carbonyl group of CHEMS 
Table S4. Average values of the total potential energy for the sitosterol-oryzanol dimer, decane, oryzanol, and sitosterol simulations, and the computed binding enthalpy $\Delta H$.

\begin{tabular}{cc}
\hline Simulation & Potential Energy $(\mathbf{K c a l} / \mathbf{m o l})$ \\
\hline Decane & 2061.8 \\
\hline Sitosterol-Oryzanol & 2438.9 \\
\hline Oryzanol & 2327.5 \\
\hline Sitosterol & 2175.3 \\
\hline $\boldsymbol{\Delta H}$ & Binding Enthalpy $(\mathbf{K c a l} / \mathbf{m o l})$ \\
\hline
\end{tabular}




\section{Figures}

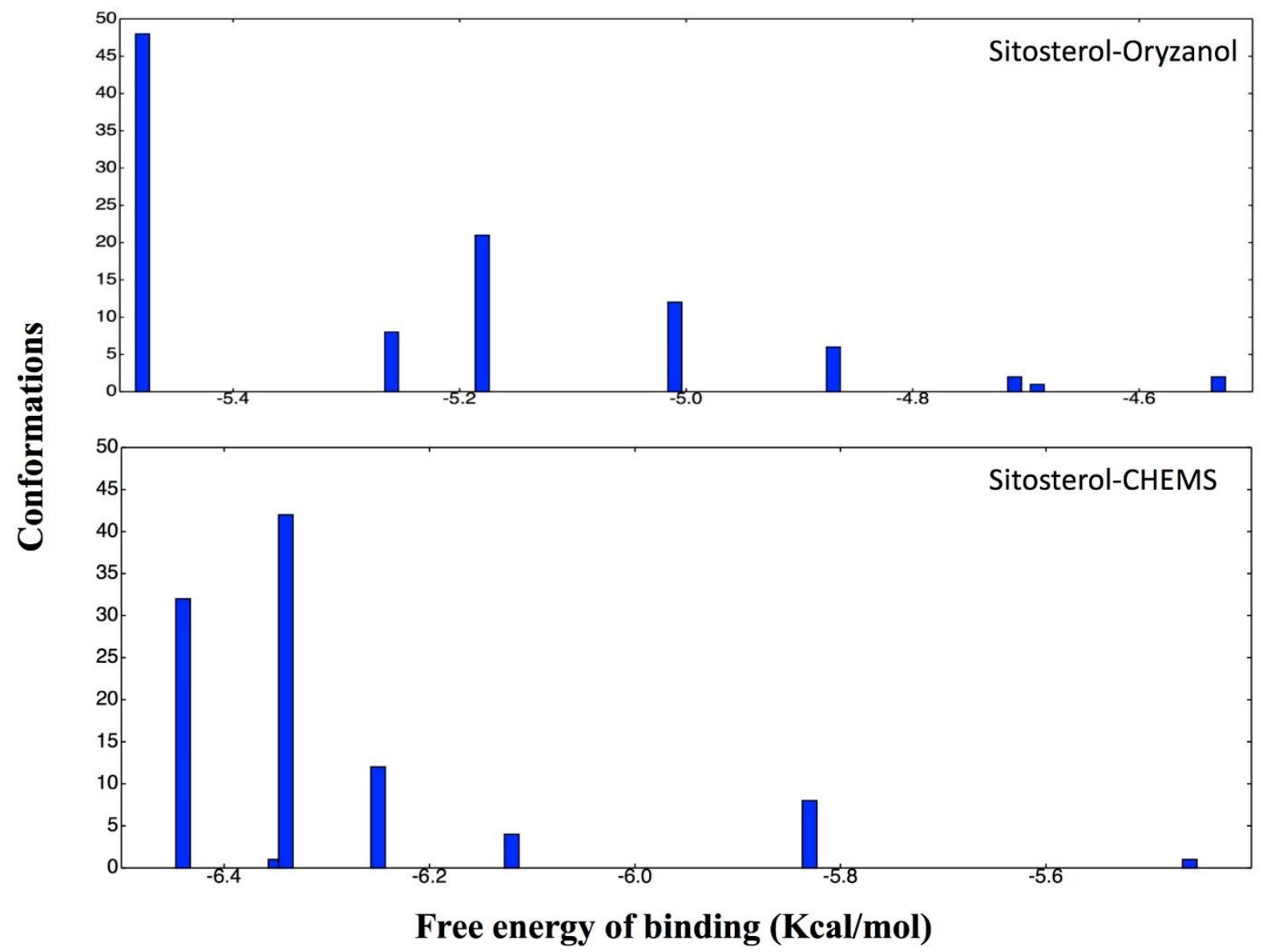

Figure S1. Histogram of the free energy of binding for the clusters of the sitosterol-oryzanol and sitosterol-CHEMS systems. Autodock clusters by first sorting all the docked conformations from lowest energy (best docking) to highest. ${ }^{8}$ The best overall docked conformation is used as the 'seed' for the first cluster. Then the coordinates of the second-best conformation are compared with those of the best to calculate the root-meansquare deviation between the two conformations. If the calculated rms value is smaller than the specified cut-off, which is $2.0 \AA$, that conformation is added to the 'bin' containing the best conformation. If not, the second becomes the reference for a second 'bin'. Then the rms between the third conformation and the 'best' is computed. If close enough, it is added to the first bin. If not it compared with the seed of the second bin and so on. 


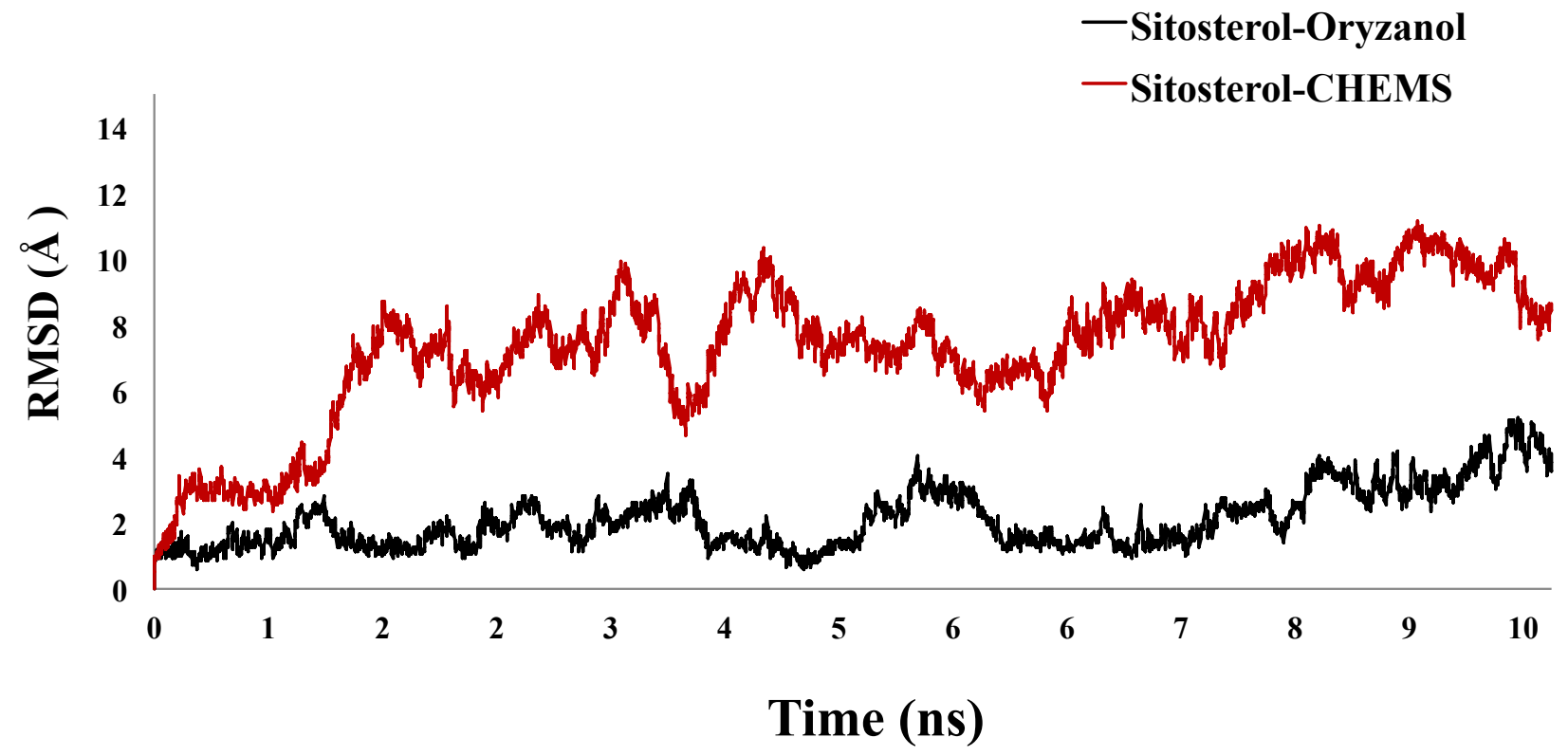

Figure S2. Time-dependent RMSD from the starting conformations for the sitosterol-oryzanol and sitosterol-CHEMS dimers.

a)

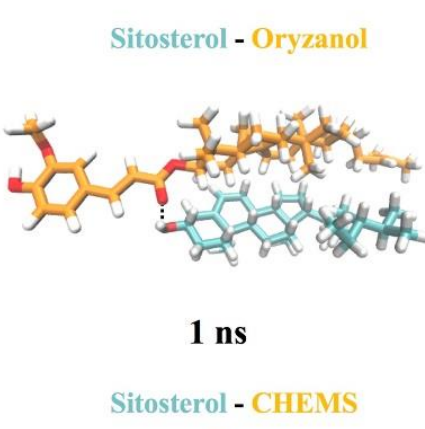

b)
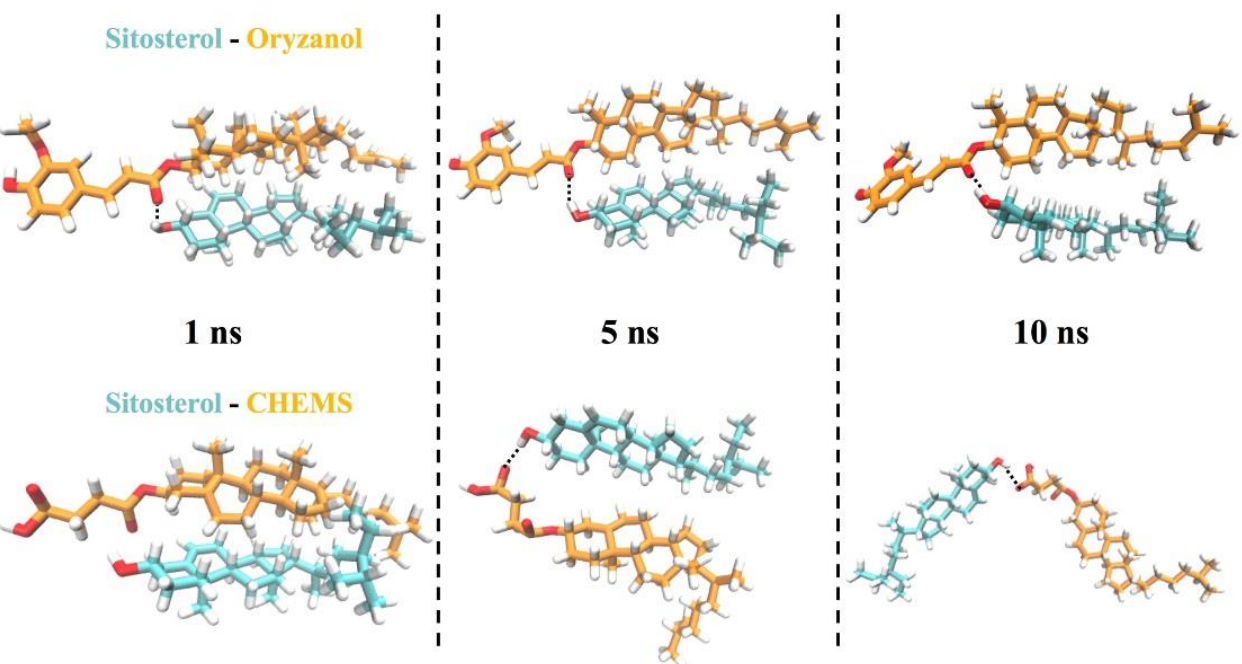

Figure S3. Snapshots of the sitosterol-oryzanol and sitosterol-CHEMS dimers during the MD simulation. Hydrogen bonds are indicated with dashed line. 

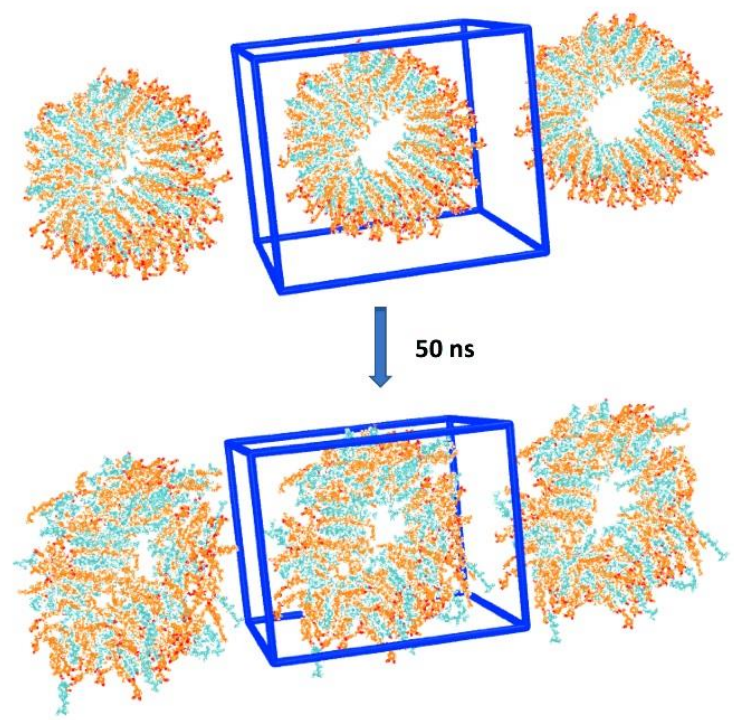

Figure S4. Snapshots of the sitosterol-oryzanol system in the primary cell (inside the box) together with two replicas. 


\section{References}

1. J. Wang, R. M. Wolf, J. W. Caldwell, P. A. Kollman and D. A. Case, J Comput Chem, 2004, 25, 1157-1174.

2. V. Hornak, R. Abel, A. Okur, B. Strockbine, A. Roitberg and C. Simmerling, Proteins, 2006, 65, 712-725.

3. R. W. Pastor, B. R. Brooks and A. Szabo, Molecular Physics, 1988, 65, 1409-1419.

4. H. J. C. Berendsen, J. P. M. Postma, W. F. v. Gunsteren, A. DiNola and J. R. Haak, The Journal of Chemical Physics, 1984, 81, 3684-3690.

5. J.-P. Ryckaert, G. Ciccotti and H. J. C. Berendsen, Journal of Computational Physics, 1977, 23, 327-341.

6. T. Darden, D. York and L. Pedersen, The Journal of Chemical Physics, 1993, 98, 10089-10092.

7. A. T. Fenley, N. M. Henriksen, H. S. Muddana and M. K. Gilson, J Chem Theory Comput, 2014, 10, 4069-4078.

8. G. M. Morris, R. Huey, W. Lindstrom, M. F. Sanner, R. K. Belew, D. S. Goodsell and A. J. Olson, J Comput Chem, 2009, 30, 2785-2791. 